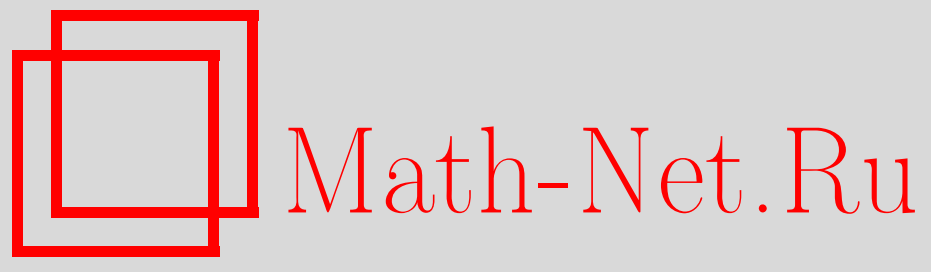

А. А. Короткевич, Интегрируемые гамильтоновы системы на алгебрах Ли малой размерности, Матем. сб., 2009, том 200, номер 12, 3-40

DOI: https://doi.org/10.4213/sm7556

Использование Общероссийского математического портала Math-Net.Ru подразумевает, что вы прочитали и согласны с пользовательским соглашением http://www . mathnet.ru/rus/agreement

Параметры загрузки:

IP: 54.162 .127 .20

26 апреля 2023 г., 15:49:38

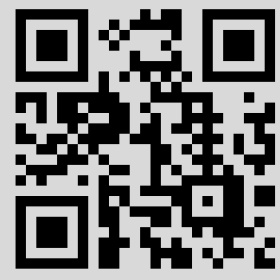




\title{
А. А. Короткевич
}

\section{Интегрируемые гамильтоновы системы на алгебрах Ли малой размерности}

\begin{abstract}
Для каждой вещественной алгебры Ли размерности три, четыре, пять и нильпотентной алгебры размерности шесть на двойственном к ней пространстве найдена интегрируемая гамильтонова система с полиномиальными первыми интегралами. Эти системы построены с помощью полного коммутативного набора полиномов на коалгебре Ли, получаемого методом С. Т. Садэтова.
\end{abstract}

Библиография: 17 названий.

Ключевые слова: интегрируемые гамильтоновы системы, полные коммутативные наборы полиномов, метод Садэтова.

\section{$\S 1$. Введение}

Как известно, на коалгебре любой конечномерной алгебры Ли существует скобка Пуассона-Ли. Набор функций на коалгебре, коммутирующих относительно этой скобки, называется полньм, если количество функций в нем равно полусумме размерности алгебры и ее индекса. Хорошо известно, что любой полный коммутативный набор функций задает вполне интегрируемую по Лиувиллю гамильтонову систему на каждой орбите коприсоединенного представления.

Настоящая работа посвящена изучению полных коммутативных наборов полиномов на коалгебрах Ли. В ней показано, что среди алгебр Ли малой размерности существуют следующие алгебры: две исключительные трехмерные, две четырехмерные, семь пятимерных, четыре шестимерных нильпотентных, на которых полный коммутативный набор состоит из линейных и квадратичных полиномов. Существует одна исключительная пятимерная алгебра Ли, на которой полный коммутативный набор состоит из кубического и линейных полиномов, причем эта алгебра является единственной неразложимой алгеброй общего типа из рассмотренных. На остальных алгебрах Ли малой размерности существует полный коммутативный набор линейных полиномов. Также установлено, что имеет место следующий интересный эффект: некоторые неизоморфные алгебры образуют семейства, зависящие от континуального параметра, однако полные коммутативные наборы полиномов на них совпадают и не зависят от параметра. На всех рассмотренных разрешимых алгебрах Ли полные коммутативные наборы состоят из линейных и квадратичных полиномов. Поэтому гипотеза о существовании на любой разрешимой алгебре Ли полного коммутативного набора из линейных и квадратичных полиномов подтверждается для алгебр Ли малой размерности. Следовательно, контрпример к этой гипотезе может существовать только для алгебр Ли больших размерностей.

(C) А. А. Короткевич, 2009 


\section{§ 2. История вопроса и основные понятия}

Пусть $\left(M^{n}, \Pi^{i j}\right)$ - пуассоново многообразие, и на нем задана гамильтонова система с гамильтонианом $H$, т.е. фиксировано векторное поле $v=\operatorname{sgrad} H$, которое в локальных координатах имеет вид

$$
v^{i}=(\operatorname{sgrad} H)^{i}=\Pi^{i j} \frac{\partial H}{\partial x^{j}} .
$$

Первыми интегралами гамильтоновой системь называются функции, которые постоянны вдоль интегральных траекторий данной системы. Хорошо известно, что функция $F$ является первым интегралом тогда и только тогда, когда она коммутирует с гамильтонианом, т.е. выполнено равенство $\{F, H\}=0$, где $\{\cdot, \cdot\}$ - скобка Пуассона, соответствующая тензору Пуассона $\Pi^{i j}$. Для того чтобы проинтегрировать гамильтонову систему, достаточно найти нужное количество функционально независимых первых интегралов. Такой набор первых интегралов существует далеко не у всех систем, а лишь у тех, которые обладают некоторой “скрытой симметрией”. Очень часто наличие “скрытой симметрии" выражается в возможности вложить данную гамильтонову систему в некоторую алгебру Ли.

Пусть $\mathfrak{g}$ - произвольная конечномерная алгебра Ли над полем $\mathbb{K}$, a $\mathfrak{g}^{*}-$ соответствующая ей коалгебра. Тогда пространство $\mathfrak{g}^{*}$ наделяется естественной структурой пуассонова многообразия при помощи скобки Пуассона-Ли. Пусть $f, g: \mathfrak{g}^{*} \rightarrow \mathbb{K}$ - произвольные гладкие функции на $\mathfrak{g}^{*}$. Тогда их дифференциалы $d_{x} f, d_{x} g$ в произвольной точке $x \in \mathfrak{g}^{*}$ можно рассматривать как элементы алгебры $\mathfrak{g}$.

ОПРЕДЕЛЕНИЕ 1. Скобкой Пуассона-Ли двух гладких функций $f$ и $g$ на $\mathfrak{g}^{*}$ называется функция

$$
\{f, g\}(x)=\left\langle x,\left[d_{x} f, d_{x} g\right]\right\rangle,
$$

где $[\cdot, \cdot]$ - коммутатор двух элементов алгебры Ли $\mathfrak{g},\langle\cdot, \cdot\rangle$ - значение ковектора на векторе.

Таким образом, тензор Пуассона $\Pi_{i j}$, соответствующий скобке Пуассона-Ли на коалгебре $\mathfrak{g}^{*}$, имеет вид $\Pi_{i j}=c_{i j}^{k} x_{k}$, где $c_{i j}^{k}$ - структурные константы алгебры Ли $\mathfrak{g}$.

Заметим, что скобка Пуассона-Ли также корректно определена на множестве всех полиномов на $\mathfrak{g}^{*}$, которые относительно данной операции образуют подалгебру $P(\mathfrak{g})$ в алгебре всех функций на $\mathfrak{g}^{*}$. Подалгебра $P(\mathfrak{g})$ называется пуассоновой алгеброй алгебры Ли $\mathfrak{g}$.

Хорошо известно, что симплектическими листами пуассонова многообразия $\left(\mathfrak{g}^{*}, c_{i j}^{k} x_{k}\right)$ являются орбиты коприсоединенного представления группы Ли $G$, соответствующей алгебре Ли $\mathfrak{g}$. Поэтому ограничение пуассоновой структуры на орбиту коприсоединенного представления группы - симплектическая форма, называемая канонической симплектической структурой или формой Кириллова (см., например, [1]).

ОПРедЕЛЕНИЕ 2. Говорят, что гамильтонова система $v=\operatorname{sgrad} H$, заданная на пуассоновом многообразии $\left(M^{n}, \Pi^{i j}\right)$ допускает вложение в алгебру Ли $\mathfrak{g}$, 
если $M^{n}$ можно отождествить с некоторым, инвариантным относительно коприсоединенного представления группы Ли $G$, соответствующей алгебре Ли $\mathfrak{g}$, подмногообразием $N \subset \mathfrak{g}^{*}$ так, что будут выполнены следующие условия:

1) поле $v$ после отождествления будет касаться орбит коприсоединенного представления группы $G$;

2) после отождествления векторное поле $v$ на $N$ будет гамильтоновым на орбитах относительно канонической симплектической структуры.

Вложение гамильтоновых систем в алгебры Ли обладает одним полезным свойством, именно благодаря которому вложенную систему часто удается проинтегрировать.

ПРЕДЛОЖЕНИЕ 1. Пусть $f: M^{n} \rightarrow N \subset \mathfrak{g}^{*}$ - вложение гамильтоновой системы на пуассоновом многообразии $\left(M^{n}, \Pi^{i j}\right)$ в алгебру Ли g. Тогда если функции $F, H: \mathfrak{g}^{*} \rightarrow \mathbb{K}$ коммутируют на всех орбитах присоединенного представления соответствующей группы Ли $G$, то функиии $F \circ f, H \circ f: M^{n} \rightarrow \mathbb{K}$ будут коммутировать на $M^{n}$.

Таким образом, интегрирование вложенной гамильтоновой системы сводится к поиску функций на коалгебре $\mathfrak{g}^{*}$, коммутирующих с образом гамильтониана при вложении. В связи с этим возникает вопрос: на любой ли коалгебре Ли $\mathfrak{g}^{*}$ существует максимально возможный набор коммутирующих функций? Более точно, интерес представляют полные коммутативные наборы.

ОПРЕДЕЛЕНИЕ 3 . Набор функции $f_{1}, \ldots, f_{k}$ на коалгебре Ли $\mathfrak{g}^{*}$ называется полным коммутативным набором, если выполнены следующие свойства:

1) функции $f_{1}, \ldots, f_{k}$ попарно коммутируют относительно скобки Пуассона-Ли, т.е. $\left\{f_{i}, f_{j}\right\}=0, i, j=1, \ldots, k$;

2) функции $f_{1}, \ldots, f_{k}$ почти всюду на $\mathfrak{g}^{*}$ функционально независимы;

3) набор $f_{1}, \ldots, f_{k}$ состоит из максимально возможного числа функций, т.е. $k=\frac{1}{2}(\operatorname{dim} \mathfrak{g}+$ ind $\mathfrak{g})$.

Легко видеть, что каждый полный коммутативный набор функций на коалгбре Ли $\mathfrak{g}^{*}$ задает на пуассоновом многообразии $\left(\mathfrak{g}^{*}, c_{i j}^{k} x_{k}\right)$ интегрируемую гамильтонову систему. Действительно, если в качестве гамильтониана взять одну функцию из полного коммутативного набора, то остальные функции набора будут функционально независимыми первыми интегралами.

В случае, когда полные наборы ищут в классе всех гладких функций, ответ на поставленный вопрос дает следующая теорема.

Теорема 1 (А. В. Браилов; см. [1]). На любом симплектическом многообразии $M^{2 n}$ существует набор из $n$ гладких, попарно коммутирующих, почти всюду функиионально независимых функций.

Следовательно, на каждой орбите коприсоединенного представления группы Ли $G$ в коалгебре Ли $\mathfrak{g}^{*}$ существует набор гладких, коммутирующих, почти всюду функционально независимых функций, количество которых равно половине размерности орбиты. Тем не менее, для приложений в механике и физике больший интерес представляют наборы, состоящие не из произвольных гладких функций, а из полиномов. В связи с этим естественно поставить вопрос 
о существовании полного коммутативного набора полиномов на произвольной коалгебре Ли.

Впервые данная проблема в виде гипотезы была сформулирована в работе [2] А. С. Мищенко и А. Т. Фоменко.

ГиПотезА 1 (А. С. Мищенко, А. Т. Фоменко; см. [2]-[4]). Пусть $\mathfrak{g}-$ вещественная или комплексная конечномерная алгебра Ли. Тогда на $\mathfrak{g}^{*}$ существует полный коммутативный набор полиномов.

Эта гипотеза была доказана авторами для случая, когда $\mathfrak{g}$ - редуктивная алгебра Ли (см. [5], [6]). Ими была предложена универсальная конструкция, позволяющая строить коммутативные наборы полиномов на алгебрах Ли, метод сдвига аргумента.

ОПРЕДЕЛЕНИЕ 4. Функция $f: \mathfrak{g}^{*} \rightarrow \mathbb{R}$ называется инвариантом коприсоединенного представления $\mathrm{Ad}^{*}: G \rightarrow \mathrm{GL}\left(\mathfrak{g}^{*}\right)$ группы Ли $G$, если

$$
f(l)=f\left(\operatorname{Ad}_{g}^{*} l\right), \quad g \in G,
$$

где $l \in \mathfrak{g}^{*}$ - произвольный фиксированный элемент коалгебры.

Множество всех инвариантов коприсоединенного представления образует кольцо $I_{\mathrm{Ad}^{*}}(G)$. Для произвольного фиксированного элемента $a \in \mathfrak{g}^{*}$ рассмотрим следующее семейство функций:

$$
\mathscr{F}_{a}=\left\{f_{\lambda}(x)=f(x+\lambda a) \mid f \in I_{\mathrm{Ad}^{*}}(G), \lambda \in \mathbb{R}\right\} .
$$

Говорят, что данное семейство получено сдвигом инвариантов на элемент $a \in \mathfrak{g}^{*}$. Оно будет коммутативным относительно скобки Пуассона-Ли, но инварианты коприсоединенного представления группы $G$ не всегда являются полиномами. Поэтому семейство $\mathscr{F}_{a}$ может содержать неполиномиальные функции. Однако, если вместо инвариантов коприсоединенного представления сдвигать коэффициенты $f_{i}(x)$ их тейлоровского разложения в точке $a$,

$$
f(a+\lambda x)=f(a)+\lambda f_{1}(x)+\lambda^{2} f_{2}(x)+\cdots,
$$

то в результате всегда получится семейство, состоящее из полиномов, поскольку все $f_{i}$-ые - полиномы. Мищенко и Фоменко доказали, что данное семейство полиномов также будет коммутативным и, более того, полным.

Теорема 2 (А. С. Мищенко, А. Т. Фоменко; см. [5], [6]). Если $\mathfrak{g}$ - nолупростая алгебра Ли, а $а \in \mathfrak{g}^{*}$ - регулярный элемент, то семейство полиномов, полученных методом сдвига инвариантов на элемент а, содержит полный коммутативный набор полиномов.

В более поздних работах А. В. Браилова, А. В. Болсинова, В. В. Трофимова, Т. А. Певцовой, О.И. Богоявленского, А.А. Архангельского, С. Сярова, Ле Нгок Тьеуена, А. М. Переломова, А. Г. Реймана, А. В. Беляева, М. Вернь, В. А. Гинзбурга полные коммутативные наборы полиномов были построены на различных сериях алгебр Ли: вещественных нильпотентных, вполне разрешимых, борелевских подалгебрах полупростых алгебр Ли, многих полупрямых 
суммах и т.д. (см., например, [7]-[10]). Полный список соответствующих результатов приведен в [1].

В 2004 г. С. Т. Садэтов доказал гипотезу Мищенко-Фоменко для случая произвольной конечномерной алгебры Ли над полем нулевой характеристики.

Теорема 3 (С. Т. Садэтов; см. [11]). Пусть $\mathfrak{g}$ - произвольная конечномерная алгебра Ли над полем нулевой характеристики. Тогда на $\mathfrak{g}^{*}$ существует полный коммутативный набор полиномов.

Чтобы непосредственно использовать эту теорему для построения полных коммутативных наборов полиномов на конкретных алгебрах Ли, ее оригинальное доказательство удобно изложить на более наглядном языке геометрических структур. В статье [12] Болсиновым была проделана большая работа по описанию конструкции Садэтова на языке пуассоновой геометрии. Для наших целей данный подход более удобен, поэтому в дальнейшем мы будем ссылаться на исходную статью [11] и на статью [12] одновременно.

Доказательство теоремы Садэтова основано на алгоритме построения полных коммутативных наборов полиномов. В этом алгоритме используется возможность сведения построения полного коммутативного набора полиномов на произвольной алгебре Ли над полем $\mathbb{K}$ к построению полного коммутативного набора полиномов на алгебре Ли меньшей размерности, но, возможно, уже над расширенным полем. Следующая лемма играет ключевую роль в доказательстве теоремы Садэтова.

Лемма 1 (С. Т. Садэтов, А. В. Болсинов; см. [11], [12]). Любая алгебра Ли $\mathfrak{g}$ над полем $\mathbb{K}$ характеристики ноль удовлетворяет одному из следующих условий:

1) $\mathfrak{g}$ имеет коммутативный идеал $\mathfrak{h}$, не являющийся одномерным центром алгебры $\mathfrak{g}$

2) $\mathfrak{g}$ имеет идеал $\mathfrak{h}_{\mathfrak{m}}$, изоморфный алгебре Гейзенберга, и при этом иентр $\mathfrak{g}$ совпадает с иентром идеала $\mathfrak{h}_{\mathfrak{m}}$;

3) $\mathfrak{g}=L \oplus \mathbb{K}$, где $L-$ полупроста;

4) g nолупроста.

Легко видеть, что в случае, когда для алгебры Ли $\mathfrak{g}$ имеют место альтернативы 3) или 4), полный коммутативный набор полиномов получается методом сдвига аргумента. Оказывается, что в случаях 1) и 2) возможна редукция к алгебре Ли меньшей размерности.

Пусть $\mathfrak{h}$ - коммутативный идеал алгебры Ли $\mathfrak{g}$, не совпадающий с одномерным центром. В силу коммутативности идеала $\mathfrak{h}$, на $\mathfrak{h}^{*}$ существует полный коммутативный набор линейных полиномов. Таким, например, является набор координат на $\mathfrak{h}^{*}$ относительно произвольного фиксированного базиса. Дополним полный коммутативный набор полиномов на $\mathfrak{h}^{*}$ до полного коммутативного набора полиномов на всей коалгебре $\mathfrak{g}^{*}$. Для этого выясним, как устроено множество функций на $\mathfrak{g}^{*}$, коммутирующих со всеми функциями на $\mathfrak{h}^{*}$. 
Присоединенное представление алгебры ad можно ограничить на идеал $\mathfrak{h}$. Обозначим это ограничение через ad $\left.\right|_{\mathfrak{h}}$. Рассмотрим двойственное к нему представление:

$$
\left(\operatorname{ad}||_{\mathfrak{h}}\right)^{*}: \mathfrak{g} \rightarrow \operatorname{gl}\left(\mathfrak{h}^{*}\right)
$$

Для каждого элемента $x \in \mathfrak{g}^{*}$ определен образ $h=\operatorname{pr}_{\mathfrak{h}^{*}}(x)$ при естественной проекции $\mathrm{pr}_{\mathfrak{h}^{*}}: \mathfrak{g}^{*} \rightarrow \mathfrak{h}^{*}$. В свою очередь, каждому элементу $h \in \mathfrak{h}^{*}$ соответствует стационарная подалгебра $\operatorname{St}(h) \subset \mathfrak{g}$ относительно представления $\left(\left.\mathrm{ad}\right|_{\mathfrak{h}}\right)^{*}$. Имеет место следующая лемма.

Лемма 2 (С. Т. Садэтов, А. В. Болсинов; см. [11], [12]). Функиия $f: \mathfrak{g}^{*} \rightarrow \mathbb{K}$ коммутирует с любой функцией $g: \mathfrak{h}^{*} \rightarrow \mathbb{K}$ тогда и только тогда, когда

$$
d f_{x} \in \operatorname{St}(h), \quad x \in \mathfrak{g}^{*} .
$$

После того, как получен критерий принадлежности функции на $\mathfrak{g}^{*}$ множеству функций, коммутирующих со всеми функциями на $\mathfrak{h}^{*}$, построим "базисные" функции в этом множестве. Рассмотрим множество всевозможных рациональных функций $\Psi: \mathfrak{h}^{*} \rightarrow \mathfrak{g}$ таких, что $\Psi(h) \in \operatorname{St}(h), h \in \mathfrak{h}^{*}$, т.е. множество всех рациональных сечений расслоения стационарных подалгебр. По каждому такому рациональному сечению построим функцию на коалгебре следующим образом:

$$
f_{\Psi}(x)=\left\langle\Psi\left(\operatorname{pr}_{\mathfrak{h}^{*}}(x)\right), x\right\rangle .
$$

Легко проверяется, что данные функции удовлетворяют условию приведенной выше леммы. Следовательно, они коммутируют с любой функций $g: \mathfrak{h}^{*} \rightarrow \mathbb{K}$, и, более того, они образуют подалгебру $L$ в алгебре Ли всех функций на $\mathfrak{g}^{*}$. Алгебра Ли $L$, вообще говоря, бесконечномерна над полем $\mathbb{K}$, но она конечномерна над расширенным полем $\mathbb{K}(\mathfrak{h})$ - полем рациональных функций на $\mathfrak{h}^{*}$.

Лемма 3 (С. Т. Садэтов, А. В. Болсинов; см. [11], [12]). Имеет место следующая формула:

$$
\operatorname{dim}_{\mathbb{K}(\mathfrak{h})} L=\operatorname{dim}_{\mathbb{K}} \operatorname{St}(h)-\operatorname{dim}_{\mathbb{K}} \mathfrak{h}+1,
$$

где $\operatorname{St}(h)$ - стационарная подалгебра элемента $h$ общего положения относительно действия $\left(\operatorname{ad}||_{\mathfrak{h}}\right)^{*}$.

Таким образом, размерность алгебры Ли $L$ над полем $\mathbb{K}(\mathfrak{h})$ строго меньше размерности первоначальной алгебры Ли $\mathfrak{g}$ над полем $\mathbb{K}$, и оказывается, что построение полного коммутативного набора полиномов на алгебре $\mathfrak{g}$ сводится к построению полного коммутативного набора полиномов на $L$.

Лемма 4 (С. Т. Садэтов, А. В. Болсинов; см. [11], [12]). Объединение полного коммутативного набора полиномов на $\mathfrak{h}^{*}$ и полного коммутативного набора полиномов на $L^{*}$ является полным коммутативным набором функций на $\mathfrak{g}^{*}$.

Заметим, что полный коммутативный набор полиномов на $L^{*}$ состоит из полиномов над расширенным полем $\mathbb{K}(\mathfrak{h})$, которые, вообще говоря, не являются полиномами над первоначальным полем $\mathbb{K}$. Тем не менее, полученный 
полный коммутативный набор функций на $\mathfrak{g}^{*}$ легко преобразовать в полный коммутативный набор полиномов. Действительно, полиномы над $\mathbb{K}(\mathfrak{h})$ будут рациональными функциями над исходным полем $\mathbb{K}$, причем знаменателями будут исключительно полиномы на $\mathfrak{h}^{*}$. Поэтому каждую рациональную функцию можно умножить на некоторый полином на $\mathfrak{h}^{*}$ так, чтобы она стала полиномом на $\mathfrak{g}^{*}$; при этом набор останется полным и коммутативным в силу того, что координаты на $\mathfrak{h}^{*}$ входят в наш полный коммутативный набор.

Таким образом, в случае, когда реализуется возможность 1), достаточно произвести "факторизацию" по коммутативному идеалу и перейти к рассмотрению алгебры рациональных сечений расслоения стационарных подалгебр над расширенным полем. Покажем, что на алгебрах Ли, у которых есть идеал, изоморфный алгебре Гейзенберга, причем центр идеала совпадает с одномерным центром всей алгебры, также существует полный коммутативный набор полиномов.

ОПРЕДЕЛЕНИЕ 5. Алгебра $\mathfrak{h}_{m}$ называется алгеброй Гейзенберга, если выполнены следующие условия:

1) как линейное пространство, $\mathfrak{h}_{m}$ является прямой суммой одномерного подпространства и $2 m$-мерного подпространства, т.е. $\mathfrak{h}_{m}=\langle z\rangle \oplus V^{2 m}$;

2) на $V^{2 m}$ задана симплектическая структура $\omega$;

3) $z$ является одномерным центром;

4) для любых $v_{1}, v_{2} \in V^{2 m}$ выполняется

$$
\left[v_{1}, v_{2}\right]=\omega\left(v_{1}, v_{2}\right) z
$$

Как и в предыдущем случае, существование полного коммутативного набора линейных полиномов на $\mathfrak{h}_{m}^{*}$ очевидно; в качестве такого набора можно взять, например, координатные функции $z, p_{1}, \ldots, p_{m}$, где $p_{1}, \ldots, p_{m}$ - базис некоторого лагранжева подпространства $V^{2 m}$. Будем дополнять полный коммутативный набор линейных полиномов на $\mathfrak{h}_{m}^{*}$ до полного коммутативного набора полиномов на всей коалгебре $\mathfrak{g}^{*}$. Для этого построим достаточное количество функций на $\mathfrak{g}^{*}$, коммутирующих со всеми функциями на $\mathfrak{h}_{m}^{*}$.

В дальнейшем нам понадобится подалгебра, существование которой гарантирует следующая лемма.

ЛЕмма 5. Существует подалгебра $\mathfrak{b} \subset \mathfrak{g}$ такая, что

$$
\mathfrak{b}+\mathfrak{h}_{m}=\mathfrak{g}, \quad \mathfrak{b} \cap \mathfrak{h}_{m}=\langle z\rangle
$$

При этом подпространство $V^{2 m} \subset \mathfrak{h}_{m}$ инвариантно относителъно присоединенного действия $\mathfrak{b} u$, кроме того, $\mathfrak{b}$ действует на $V$ симплектическими преобразованиями.

По каждому элементу $\beta \in \mathfrak{b}$ построим квадратичный полином $f_{\beta}: \mathfrak{g}^{*} \rightarrow \mathbb{K}$ следующим образом:

$$
f_{\beta}(x)=\langle\beta, x\rangle\langle z, x\rangle+\frac{1}{2}\left\langle\omega^{-1}\left(\left(\operatorname{ad}_{\beta}\right)^{*} \operatorname{pr}_{V}(x)\right), x\right\rangle,
$$


где $x$ - произвольный элемент из $\mathfrak{g}^{*}, \operatorname{pr}_{V}: \mathfrak{g}^{*} \rightarrow V^{*}-$ проекция, $\left(\operatorname{ad}_{\beta}\right)^{*}: V^{*} \rightarrow V^{*}$ - оператор, двойственный к оператору $\operatorname{ad}_{\beta}: V \rightarrow V, \omega-$ симплектическая структура на $V$, рассматриваемая как отображение из $V$ в $V^{*}, \omega^{-1}: V^{*} \rightarrow V$ - обратный оператор. Данные квадратичные полиномы обладают требуемым свойством.

Лемма 6 (С. Т. Садэтов, А. В. Болсинов; см. [11], [12]). Многочлен $f_{\beta}(x)$ на $\mathfrak{g}^{*}$ для любого $\beta \in \mathfrak{b}$ коммутирует со всеми функииями на $\mathfrak{h}_{m}^{*}$.

Несложно показать, что многочлены $f_{\beta}$ образуют алгебру Ли, изоморфную подалгебре $\mathfrak{b}$.

Предположим, что на $\mathfrak{b}^{*}$ найден полный коммутативный набор полиномов. Тогда каждая функция из данного набора является полиномом от координат $\beta_{1}, \ldots, \beta_{s}$ на $\mathfrak{b}^{*}$ относительно некоторого фиксированного базиса $\left(s=\operatorname{dim} \mathfrak{b}^{*}\right)$. Координаты $\beta_{1}, \ldots, \beta_{s}$ можно рассматривать как элементы подалгебры $\mathfrak{b}$. Поэтому, заменив в каждом полиноме из полного коммутативного набора на $\mathfrak{b}^{*}$ все $\beta_{i}, i=1, \ldots, s$, на соответствующие им квадратичные полиномы $f_{\beta_{i}}, i=$ $1, \ldots, s$, мы получим набор полиномов на исходной коалгебре $\mathfrak{g}^{*}$.

Лемма 7 (С. Т. Садэтов, А. В. Болсинов; см. [11], [12]). Объединение полного коммутативного набора линейных полиномов на $\mathfrak{h}_{m}^{*}$ и полного коммутативного набора полиномов на $\mathfrak{b}^{*}$, в котором все $\beta_{i} \in \mathfrak{b}$ заменены на соответствующие им квадратичные полиномы $f_{\beta_{i}}$, является полным коммутативным набором полиномов на первоначальной коалгебре $\mathfrak{g}^{*}$.

Таким образом, построение полного коммутативного набора полиномов на первоначальной алгебре Ли $\mathfrak{g}$ можно свести к построению полного коммутативного набора полиномов на алгебре $\mathfrak{b}$, а так как $\operatorname{dim} \mathfrak{b}<\operatorname{dim} \mathfrak{g}$, то это и есть редукция к алгебре Ли меньшей размерности.

Таким образом, если для алгебры Ли g реализуются возможности 1) или 2), то построение полного коммутативного набора полиномов сводится к построению полного коммутативного набора полиномов на алгебре Ли меньшей размерности, но, возможно, над расширенным полем. Следовательно, на любой конечномерной алгебре Ли над полем нулевой характеристики полный коммутативный набор полиномов существует.

\section{§ 3. Полные наборы на алгебрах Ли малой размерности}

В дальнейшем нам понадобится запись части конструкции Садэтова в явной координатной форме.

Пусть алгебра $\mathfrak{g}$ имеет коммутативный идеал $\mathfrak{h}$, не совпадающий с одномерным центром. Предположим, что $\operatorname{dim} \mathfrak{g}=n, \operatorname{dim} \mathfrak{h}=k$. Выберем в алгебре $\mathfrak{g}$ базис

$$
e_{1}, \ldots, e_{k}, f_{k+1}, \ldots, f_{n}
$$


так, чтобы векторы $e_{1}, \ldots, e_{k}$ образовывали базис в идеале $\mathfrak{h}$. Тогда в этом базисе структурные константы алгебры $\mathfrak{g}$ будут выглядеть следующим образом:

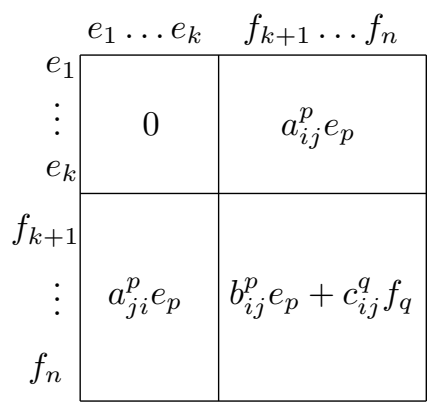

тензоры $a_{i j}^{p}, b_{i j}^{p}, c_{i j}^{q}$ кососимметричны по нижним индексам и ими задается коммутатор в алгебре $\mathfrak{g}, 1 \leqslant p \leqslant k, k+1 \leqslant q \leqslant n$. Для тензора $a_{i j}^{p}: 1 \leqslant i \leqslant k$ и $k+1 \leqslant j \leqslant n$ или $k+1 \leqslant i \leqslant n$ и $1 \leqslant j \leqslant k$. Для тензоров $b_{i j}^{p}$ и $c_{i j}^{q}: k+1 \leqslant i \leqslant n$ и $k+1 \leqslant j \leqslant n$.

Так как $\mathfrak{h}$ - идеал, то присоединенное представление ad можно ограничить на $\mathfrak{h}$. Рассмотрим ограничение ad $\left.\right|_{\mathfrak{h}}$. При таком представлении каждому элементу алгебры $\mathfrak{g}$ сопоставим линейный оператор на $\mathfrak{h}$. Посмотрим, какие операторы при этом соответствуют базисным элементам. Так как идеал $\mathfrak{h}$ коммутативный, то элементам $e_{1}, \ldots, e_{k}$ соответствуют тождественно нулевые операторы на $\mathfrak{h}$, а каждому элементу $f_{m}$ соответствует оператор, который в данном базисе задается матрицей $\left(a_{m j}^{i}\right)$.

Пусть $\xi_{1}, \ldots, \xi_{k}, \eta_{k+1}, \ldots, \eta_{n}-$ базис в коалгебре $\mathfrak{g}^{*}$, двойственный к базису $e_{1}, \ldots, e_{k}, f_{k+1}, \ldots, f_{n}$. Рассмотрим представление $\left(\left.\operatorname{ad}\right|_{\mathfrak{h}}\right)^{*}$, двойственное к представлению ad $\left.\right|_{\mathfrak{h}}$. При таком представлении каждому элементу алгебры будет соответствовать оператор на $\mathfrak{h}^{*}$. Какие операторы будут соответствовать базисным элементам алгебры $\mathfrak{g}$ ? Элементам $e_{1}, \ldots, e_{k}$ будут соответствовать нулевые операторы на $\mathfrak{h}^{*}$, а каждому элементу $f_{m}$ будет соответствовать оператор, который в базисе $\xi_{1}, \ldots, \xi_{k}$ задается матрицей $\left(a_{m j}^{i}\right)^{*}$.

Теперь выясним, как устроены рациональные сечения $\Psi: \mathfrak{h}^{*} \rightarrow \mathfrak{g}$ такие, что

$$
\Psi(h) \in \operatorname{St}_{(\operatorname{ad} \mid \mathfrak{h})^{*}}(h), \quad h \in \mathfrak{h}^{*} .
$$

Зафиксируем некоторый элемент $h \in \mathfrak{h}^{*}$ и найдем его стабилизатор. Пусть $h=\sum_{i=1}^{k} h_{i} \xi_{i}$. Рассмотрим произвольный элемент $x \in \mathfrak{g}$. Пусть

$$
x=\sum_{i=1}^{k} x_{i} e_{i}+\sum_{i=k+1}^{n} x_{i} f_{i} .
$$

Найдем действие образа элемента $x$ на $h$ при представлении $\left(\left.\operatorname{ad}\right|_{\mathfrak{h}}\right)^{*}$. Обозначим через $A_{x}$ оператор, соответствующий элементу $x$ при представлении $\left(\left.\operatorname{ad}\right|_{\mathfrak{h}}\right)^{*}$. Тогда

$$
x \in \mathrm{St}_{(\operatorname{ad} \mid \mathfrak{h})^{*}}(h) \quad \Longleftrightarrow \quad A_{x}(h)=0 \in \mathfrak{h}^{*}
$$


т.е. $\left\langle A_{x}(h), y\right\rangle=0, y \in \mathfrak{h}$. Это равносильно тому, что $\left\langle A_{x}(h), e_{i}\right\rangle=0$, где $i=1, \ldots, k$. Теперь можно найти стабилизатор $\operatorname{St}_{(\operatorname{ad} \mid \mathfrak{h})^{*}}(h)$ :

$$
\left\langle A_{x}(h), e_{i}\right\rangle=\sum_{j=1}^{k} x_{j}\left\langle A_{e_{j}}(h), e_{i}\right\rangle+\sum_{j=k+1}^{n} x_{j}\left\langle A_{f_{j}}(h), e_{i}\right\rangle=\sum_{j=k+1}^{n} x_{j}\left\langle A_{f_{j}}(h), e_{i}\right\rangle .
$$

Так как $h=\sum_{p=1}^{k} h_{p} \xi_{p}$, то

$$
\sum_{j=k+1}^{n} x_{j}\left\langle A_{f_{j}}(h), e_{i}\right\rangle=\sum_{j=k+1}^{n} x_{j} \sum_{p=1}^{k} h_{p}\left\langle A_{f_{j}}\left(\xi_{p}\right), e_{i}\right\rangle .
$$

Выше отмечалось, что $A_{f_{m}}=\left(a_{m j}^{i}\right)^{*}$. Поэтому

$$
\left\langle A_{f_{j}}\left(\xi_{p}\right), e_{i}\right\rangle=\left\langle\xi_{p}, A_{f_{j}}^{*}\left(e_{i}\right)\right\rangle=\left\langle\xi_{p}, a_{j i}^{s} e_{s}\right\rangle=a_{j i}^{p} .
$$

Значит, стабилизатор элемента $h=\sum_{p=1}^{k} h_{p} \xi_{p}$ задается следующей системой уравнений относительно переменных $x_{j}$ :

$$
\sum_{j=k+1}^{n} \sum_{p=1}^{k} x_{j} h_{p} a_{j i}^{p}=0, \quad i=1, \ldots, k .
$$

Отметим, что в данной системе отсутствуют неизвестные $x_{1}, \ldots, x_{k}$. Поэтому размерность стабилизатора любого элемента $h$ всегда больше или равна $k$. Пусть $\operatorname{dim}_{\mathbb{K}} \operatorname{St}(h)=l, l \geqslant k$. Тогда $l$ неизвестных (без потери общности можно считать, что это $x_{1}, \ldots, x_{k}, x_{k+1}, \ldots, x_{l}$ ) образуют фундаментальную систему решений. Поэтому остальные неизвестные: $x_{l+1}, \ldots, x_{n}$ выражаются через них линейно, т.е.

$$
\left\{\begin{array}{l}
x_{l+1}=c_{l+1, k+1} x_{k+1}+\cdots+c_{l+1, l} x_{l}, \\
\vdots \\
x_{n}=c_{n, k+1} x_{k+1}+\cdots+c_{n, l} x_{l},
\end{array}\right.
$$

где $c_{i, j}$ - рациональные функции от $h_{1}, \ldots, h_{k}$.

Таким образом, каждое рациональное сечение расслоения стационарных подалгебр есть набор из $n$ рациональных функций $x_{1}, \ldots, x_{n}$ :

$$
\left(h_{1}, h_{2}, \ldots, h_{k}\right) \stackrel{\Psi}{\longmapsto} \underbrace{\left(x_{1}, \ldots, x_{k}, x_{k+1}, \ldots, x_{l}\right.}_{\text {произвольные }}, \underbrace{\left.x_{l+1}, \ldots, x_{n}\right)}_{\text {из системы }(*)}
$$

причем в качестве функций $x_{1}, \ldots, x_{l}$ можно взять произвольные рациональные функции от $h_{1}, \ldots, h_{k}$, а функции $x_{l+1}, \ldots, x_{n}$ будут их линейными комбинациями с коэффициентами $c_{i, j}$ согласно системе $(*)$. Как отмечалось выше, коэффициенты $c_{i, j}$ в системе $(*)$ - рациональные функции от $h_{1}, \ldots, h_{k}$, поэтому $x_{l+1}, \ldots, x_{n}$ также будут рациональными функциями от $h_{1}, \ldots, h_{k}$. Зафиксируем любое рациональное сечение. Для этого достаточно задать следующие рациональные функции:

$$
x_{1}=R_{1}\left(h_{1}, \ldots, h_{k}\right), \quad \ldots, \quad x_{l}=R_{l}\left(h_{1}, \ldots, h_{k}\right) .
$$


Зная $x_{1}, \ldots, x_{l}$, находим

$$
x_{l+1}=L_{l+1}\left(R_{k+1}, \ldots, R_{l}\right), \quad \ldots, \quad x_{n}=L_{n}\left(R_{k+1}, \ldots, R_{l}\right) ;
$$

определение линейных функций $L_{l+1}, \ldots, L_{n}$ содержится в системе $(*)$. Согласно общей конструкции необходимо по каждому рациональному сечению построить функцию на $\mathfrak{g}^{*}$ следующим образом:

$$
f_{\Psi}(\zeta)=\left\langle\Psi\left(\operatorname{pr}_{\mathfrak{h}^{*}}(\zeta)\right), \zeta\right\rangle, \quad \zeta \in \mathfrak{g}^{*}
$$

Пусть

$$
\zeta=\sum_{i=1}^{k} \zeta_{i} \xi_{i}+\sum_{i=k+1}^{n} \zeta_{i} \eta_{i} .
$$

Тогда $\operatorname{pr}_{\mathfrak{h} *}(\zeta)=\sum_{i=1}^{k} \zeta_{i} \xi_{i}$. Имеем

$$
\begin{gathered}
\Psi\left(\operatorname{pr}_{\mathfrak{h}^{*}}(\zeta)\right)=\left(R_{1}\left(\zeta_{1}, \ldots, \zeta_{k}\right), \ldots, R_{l}\left(\zeta_{1}, \ldots, \zeta_{k}\right),\right. \\
\left.L_{l+1}\left(R_{k+1}, \ldots, R_{l}\right), \ldots, L_{n}\left(R_{k+1}, \ldots, R_{l}\right)\right) \\
f_{\Psi}(\zeta)=\left\langle\Psi\left(\operatorname{pr}_{\mathfrak{h}^{*}}(\zeta)\right), \zeta\right\rangle=R_{1}\left(\zeta_{1}, \ldots, \zeta_{k}\right) \zeta_{1}+\cdots+R_{l}\left(\zeta_{1}, \ldots, \zeta_{k}\right) \zeta_{l} \\
+L_{l+1}\left(R_{k+1}, \ldots, R_{l}\right) \zeta_{l+1}+\cdots+L_{n}\left(R_{k+1}, \ldots, R_{l}\right) \zeta_{n} \\
=R_{1} \zeta_{1}+\cdots+R_{l} \zeta_{l}+\left(c_{l+1, k+1} R_{k+1}+\cdots+c_{l+1, l} R_{l}\right) \zeta_{l+1}+\cdots \\
\quad+\left(c_{n, k+1} R_{k+1}+\cdots+c_{n, l} R_{l}\right) \zeta_{n} \\
=R_{1} \zeta_{1}+\cdots+R_{k} \zeta_{k}+R_{k+1}\left(\zeta_{k+1}+c_{l+1, k+1} \zeta_{l+1}+\cdots+c_{n, k+1} \zeta_{n}\right)+\cdots \\
+R_{l}\left(\zeta_{l}+c_{l+1, l} \zeta_{l+1}+\cdots+c_{n, l} \zeta_{n}\right) .
\end{gathered}
$$

Очевидно, что любая функция, построенная по рациональному сечению, представляется в виде суммы элемента поля $\mathbb{K}(\mathfrak{h})$ (первые $k$ слагаемых) и линейной комбинации с коэффициентами из $\mathbb{K}(\mathfrak{h})$ функций

$$
\varphi_{i}=\zeta_{i}+c_{l+1, i} \zeta_{l+1}+\cdots+c_{n, i} \zeta_{n} .
$$

Рассмотрим следующие сечения:

$$
\begin{aligned}
\Psi_{0}:\left\{\begin{array}{l}
x_{1}=1, \\
x_{2}=0, \\
\vdots \\
x_{k}=0,
\end{array}\right. & \Psi_{i}:\left\{\begin{array}{l}
x_{1}=0, \\
\vdots \\
x_{k}=0,
\end{array}\right. \\
\left\{\begin{array}{l}
x_{k+1}=0, \\
\vdots \\
x_{n}=0,
\end{array}\right. & \left\{\begin{array}{l}
x_{k+1}=0, \\
\vdots \\
x_{i}=1, \\
\vdots \\
x_{l}=0,
\end{array}\right. \\
& \left\{\begin{array}{l}
x_{l+1}=c_{l+1, i}, \\
\vdots \\
x_{n}=c_{n, i},
\end{array}\right.
\end{aligned}
$$


При гомоморфизме $\Psi \rightarrow f_{\Psi}$ сечение $\Psi_{0}$ переходит в элемент поля $\mathbb{K}(\mathfrak{h})$, который мы обозначим через $\varphi_{0}$, а сечения $\Psi_{i}$ переходят в функции $\varphi_{i}$. Функции $\varphi_{0}, \varphi_{k+1}, \ldots, \varphi_{l}$ образуют базис в алгебре $L$.

Теперь перейдем к построению полных коммутативных наборов полиномов.

В настоящей работе для всех вещественных алгебр Ли малой размерности, a точнее, для всех алгебр Ли размерности три, четыре, пять и нильпотентных алгебр размерности шесть построена интегрируемая гамильтонова система на коалгебре с полиномиальными первыми интегралами. Для построения такой системы на каждой алгебре Ли найден полный коммутативный набор полиномов методом Садэтова. В работе использована классификация неразложимых (т.е. непредставимых в виде прямой суммы) алгебр Ли малой размерности, приведенная в статье [13]. Помимо этого рассмотрены все разложимые алгебры Ли малой размерности, которые классифицированы в работах В. В. Морозова (см. [14]) и Г. М. Мубаракзянова (см. [15]).

Полная классификация алгебр Ли малой размерности состоит из ста сорока алгебр. Среди них одиннадцать трехмерных алгебр (девять неразложимых и две разложимые), двадцать четыре четырехмерных (двенадцать неразложимых и двенадцать разложимых), семьдесят три пятимерных (сорок неразложимых и тридцать три разложимых), тридцать две шестимерные нильпотентные (двадцать две неразложимые и десять разложимых). Коммутационные соотношения некоторых алгебр зависят от континуального параметра, причем при различных значениях параметра алгебры будут неизоморфны. Таким образом, некоторые алгебры по сути представляют собой целое семейство неизоморфных алгебр, зависящих от континуального параметра. Во всех рассмотренных алгебрах зависимость коммутационных соотношений от параметра линейная. Количество таких семейств равно сорока одному. Два семейства трехмерны и неразложимы (т.е. каждая алгебра из данного семейства неразложима), пять четырехмерных неразложимых, два четырехмерных разложимых, двадцать одно пятимерное неразложимое, девять пятимерных разложимых, два шестимерных неразложимых.

Среди рассматриваемых нами алгебр Ли есть алгебры различных типов, а именно, четыре абелевы, сорок две нильпотентные, восемьдесят пять разрешимых, две полупростые, четыре редуктивные.

Придерживаясь обозначений, использованных в статье [13], мы каждой алгебре поставим в соответствие символ $A_{n, m}$, где $n$ - размерность алгебры, a $m$ - ее порядковый номер в классификации алгебр той же размерности, причем иногда одним символом будем обозначать целое семейство алгебр, зависящее от континуального параметра.

Алгебры Ли малой размерности можно разбить на семь классов таких, что для всех алгебр из одного класса метод Садэтова реализуется одинаково (метод Садэтова, по сути, является алгоритмом, и на каждом шаге этого алгоритма есть несколько возможностей; мы говорим, что метод Садэтова для двух алгебр реализуется одинаково, если на каждом шаге для этих алгебр выбираются одинаковые возможности). Заметим, что полученное разбиение всех алгебр Ли малой размерности на классы никак не коррелирует с разбиениями, которые 
задают размерность алгебры или ее тип. Таким образом, принадлежность алгебры некоторому классу является особой характеристикой ее внутренней структуры.

Результаты построения полных коммутативных наборов на вышеуказанных алгебрах приведен в таблицах 1-4. Каждая таблица состоит из пяти столбцов. В первом столбце указан символ данной алгебры и ее класс. Во втором столбце содержатся все ненулевые коммутаторы базисных элементов алгебры, при этом прочерк означает, что таких коммутаторов нет, т.е. алгебра абелева. В третьем столбце указан тип алгебры: полупростая, редуктивная, нильпотентная, разрешимая, абелева или алгебра общего типа, если ее тип не совпадает ни с одним из перечисленных. В четвертом столбце указаны инварианты алгебры; в случае, когда рассматривается не одна алгебра, а сразу целое семейство, зависящее от параметра, также указаны ограничения на параметр (если для параметра не указаны никакие ограничения, то считается, что он может принимать любое вещественное значение). В пятом столбце указан построенный набор полиномов, задающих интегрируемую гамильтонову систему на коалгебре с полиномиальными первыми интегралами (полный набор).

В четвертом столбце указаны инварианты, найденные в работе [13]. Отметим, что некоторые из приведенных инвариантов представляют собой комплекснозначные функции, тем не менее все такие комплекснозначные функции имеют вид $(\bar{z} / z)^{i a}$, где $z$ - некоторое комплексное число, $\bar{z}$ - сопряженное ему число, $a$ - вещественное число. Поэтому легко видеть, что эти функции являются вещественнозначными.

В работе [13] также были обнаружены следующие опечатки.

1) В алгебре $A_{4,5}$ неправильно указаны условия на параметры: вместо $-1 \leqslant$ $a \leqslant b \leqslant 1$ должно быть $-1 \leqslant b \leqslant a \leqslant 1$.

2) У алгебры $A_{5,29}$ неверно написаны коммутационные соотношения: вместо

$$
\left[e_{2}, e_{4}\right]=e_{1}, \quad\left[e_{1}, e_{5}\right]=e_{1}, \quad\left[e_{2}, e_{5}\right]=e_{2}, \quad\left[e_{4}, e_{5}\right]=e_{3}
$$

должно быть

$$
\left[e_{2}, e_{3}\right]=e_{1}, \quad\left[e_{1}, e_{5}\right]=e_{1}, \quad\left[e_{2}, e_{5}\right]=e_{2}, \quad\left[e_{3}, e_{5}\right]=e_{4} .
$$

Следовательно, и инвариант найден неправильно: вместо $x_{3}$ должен быть $x_{4}$.

3) В статье написано, что алгебра $A_{5,40}$ разрешима. На самом деле она неразрешима и является алгеброй общего типа.

4) Неверно указан диапазон для параметра $а$ в алгебрах $A_{6,5}$ и $A_{6,10}$. Вместо $a \neq 0$ должно быть $a= \pm 1$ : так как при всех $a>0$ и при всех $a<0$ алгебры будут изоморфны, то нужно взять одно положительное и одно отрицательное значение $a$.

5) Неправильно найдены инварианты на алгебрах $A_{5,15}$ : вместо

$$
\frac{x_{1}^{a}}{x_{3}}, \quad x_{1} \exp \left(-\frac{x_{2}}{x_{1}}\right), \quad x_{3} \exp \left(-\frac{x_{4}}{x_{3}}\right)
$$

должно быть

$$
\frac{x_{1}^{a}}{x_{3}}, \quad x_{1} \exp \left(-\frac{x_{2}}{x_{1}}\right), \quad x_{3} \exp \left(-\frac{a x_{4}}{x_{3}}\right)
$$


на алгебрах $A_{5,16}$ вместо

$$
\frac{x_{1}^{2 p}}{x_{3}^{2}+x_{4}^{2}}, \quad x_{1}^{2 q} \frac{x_{3}-i x_{4}}{x_{3}+i x_{4}}, \quad x_{1} \exp \left(-\frac{x_{2}}{x_{1}}\right)
$$

должно быть

$$
\frac{x_{1}^{2 p}}{x_{3}^{2}+x_{4}^{2}}, \quad x_{1}^{2 q}\left(\frac{p x_{3}-q x_{4}-i\left(p x_{4}+q x_{3}\right)}{p x_{3}-q x_{4}+i\left(p x_{4}+q x_{3}\right)}\right)^{-i}, \quad x_{1} \exp \left(-\frac{x_{2}}{x_{1}}\right) .
$$

Эта неточность, а также правильные инварианты были найдены Балиной в работе [16].

В приведенных ниже таблицах указанные выше опечатки устранены. Таким образом, в настоящей работе, в частности, содержится выверенный список всех алгебр Ли малой размерности и их инвариантов.

Найденные полные коммутативные наборы полиномов были использованы Зуевым в диссертации [17] при изучении формального метода сдвига аргумента для алгебр Ли над произвольным полем характеристики нуль.

Перейдем к подробному рассмотрению каждого из семи классов алгебр.

Класс I. Полупростые и редуктивные алгебры. Среди рассматриваемых нами алгебр есть две полупростые, а именно, $A_{3,8}$ (это в точности алгебра $\mathfrak{s u}(1,1))$ и $A_{3,9}$ (это в точности алгебра $\left.\mathfrak{s u}(2)\right)$, и четыре редуктивные:

$$
A_{4,23}, \quad A_{4,24}, \quad A_{5,51}, \quad A_{5,52} .
$$

УтвЕРЖДЕНИЕ 1. На всех алгебрах Ли класса I существует интегрируемая гамильтонова система с полиномиальными первыми интегралами (см. таблицы 1-4, где в первом столбце - символы, во втором - коммутационнье соотношения, в третьем - типь алгебр, в четвертом - инварианты, а в пятом - полные наборы полиномиальных интегралов).

Для построения на алгебре Ли интегрируемой гамильтоновой системы с полиномиальными первыми интегралами достаточно найти полный коммутативный набор полиномов на данной алгебре. Будем строить полный коммутативный набор полиномов по алгоритму Садэтова. Для полупростых и редуктивных алгебр он совпадает с методом сдвига аргумента (этот метод описан в работах Мищенко и Фоменко [5], [6]), поэтому здесь мы не будем приводить построение полного коммутативного набора.

\section{Класс II. Алгебры, в которых существует большой коммутативный} идеал. Среди рассматриваемых нами алгебр есть сто шестнадцать алгебр, в которых существует большой коммутативный идеал, т.е. такой коммутативный идеал, что его базис (рассматриваемый как набор линейных полиномов) 
образует полный коммутативный набор на всей алгебре. Эти алгебры таковы:

$$
\begin{array}{ccccc}
A_{3,1}-A_{3,7} ; & A_{3,10} ; \quad A_{3,11} ; & \\
A_{4,1}-A_{4,7} ; \quad A_{4,9} ; \quad A_{4,12}-A_{4,22} ; & \\
A_{5,1} ; \quad A_{5,2} ; & A_{5,4}-A_{5,24} ; & A_{5,27}-A_{5,35} ; \quad A_{5,38} ; & A_{5,39} ; \\
A_{5,41}-A_{5,50} ; & A_{5,53}-A_{5,59} ; & A_{5,62}-A_{5,68} ; & A_{5,70} ; & A_{5,73} ; \\
A_{6,1} ; & A_{6,2} ; & A_{6,4}-A_{6,20} ; & A_{6,23}-A_{6,28} ; & A_{6,30}-A_{6,32} .
\end{array}
$$

УтВЕРЖДЕНИЕ 2. На всех алгебрах Ли класса II явно указана интегрируемая гамильтонова система с полиномиальными первыми интегралами (см. таблиць 1-4).

Для построения на алгебре Ли интегрируемой гамильтоновой системы с полиномиальными первыми интегралами достаточно найти полный коммутативный набор полиномов на данной алгебре. Будем строить полный коммутативный набор полиномов по алгоритму Садэтова. Для алгебр класса II алгоритм Садэтова будет состоять из следующих шагов:

1) В первоначальной алгебре Ли есть коммутативный идеал, не являющийся одномерным центром, поэтому базисные элементы идеала образуют часть полного коммутативного набора. Для нахождения оставшейся части полного коммутативного набора необходимо рассмотреть алгебру рациональных сечений.

2) Поскольку коммутативный идеал имеет большую размерность, алгебра рациональных сечений одномерна и, следовательно, коммутативна. Поэтому полный коммутативный набор полиномов на ней строится тривиально.

Рассмотрим разрешимую алгебру $A_{3,4}$.

$A_{3,4}:$\begin{tabular}{|c|c|c|}
\hline 0 & 0 & $e_{1}$ \\
\hline 0 & 0 & $-e_{2}$ \\
\hline$-e_{1}$ & $e_{2}$ & 0 \\
\hline
\end{tabular}

Eе размерность $\operatorname{dim} A_{3,4}=3$, а индекс ind $A_{3,4}=1$, поэтому полный коммутативный набор должен состоять из $k=(3+1) / 2=2$ полиномов. Легко видеть, что в данной алгебре существует коммутативный идеал, натянутый на векторы $e_{1}$ и $e_{2}$. Поэтому координаты на идеале, рассматриваемые как линейные полиномы на коалгебре, будут образовывать часть полного коммутативного набора. Далее переходим к рассмотрению алгебры рациональных сечений расслоения стационарных подалгебр. В силу того, что идеал достаточно большой, она оказывается одномерной, поэтому координат на идеале уже хватает для полного коммутативного набора полиномов на всей алгебре.

Для остальных алгебр данного класса построения интегралов и соответствующие вычисления проводятся аналогично, и мы их опускаем. Полученные результаты приведены в таблицах 1-4.

Класс III. Алгебры, в которых существует коммутативный идеал, а алгебра рациональных сечений двумерна. Следующий класс алгебр, к рассмотрению которого мы переходим, состоит из алгебр, в которых существует коммутативный идеал (не являющийся одномерным центром), а алгебра 
рациональных сечений расслоения стационарных подалгебр над расширенным полем двумерна. Этот класс состоит из девяти алгебр: $A_{4,8}, A_{5,3}, A_{5,36}, A_{5,40}$, $A_{5,69}, A_{6,3}, A_{6,21}, A_{6,22}, A_{6,29}$.

УТВЕРЖДЕНИЕ 3. На всех алгебрах Ли класса III явно указана интегрируемая гамильтонова система с полиномиальными первыми интегралами (см. таблицьь 1-4).

Для построения на алгебре Ли интегрируемой гамильтоновой системы с полиномиальными первыми интегралами достаточно найти полный коммутативный набор полиномов на данной алгебре. Будем строить полный коммутативный набор полиномов по алгоритму Садэтова. Для алгебр класса III алгоритм Садэтова будет состоять из следующих шагов:

1) В первоначальной алгебре Ли есть коммутативный идеал, не являющийся одномерным центром, поэтому базисные элементы идеала образуют часть полного коммутативного набора. Для нахождения оставшейся части полного коммутативного набора необходимо рассмотреть алгебру рациональных сечений.

2) Алгебра рациональных сечений двумерна, но так как у нее всегда есть центр, то она коммутативна. Поэтому полный коммутативный набор полиномов на ней строится тривиально, в качестве него можно взять базисные элементы алгебры сечений.

3) Так как алгебра рациональных сечений рассматривалась над расширенным полем, то некоторые из полученных полиномов над первоначальным полем могут оказаться рациональными функциями. Умножая рациональные функции на соответствующие знаменатели, получаем полный коммутативный набор полиномов над исходным полем.

Рассмотрим разрешимую алгебру $A_{4,8}$.

\begin{tabular}{|c|c|c|c|c|}
\hline \multirow{4}{*}{$A_{4,8}:$} & 0 & 0 & 0 & 0 \\
\hline & 0 & 0 & $e_{1}$ & $e_{2}$ \\
\hline & 0 & $-e_{1}$ & 0 & $-e_{3}$ \\
\hline & 0 & $-e_{2}$ & $e_{3}$ & 0 \\
\hline
\end{tabular}

Eе размерность $\operatorname{dim} A_{4,8}=4$, а индекс ind $A_{4,8}=2$, поэтому полный коммутативный набор должен содержать $k=(4+2) / 2=3$ полинома. В этой алгебре существует коммутативный идеал, он натянут на векторы $e_{1}$ и $e_{2}$.

Согласно методу Садэтова, теперь необходимо перейти к алгебре рациональных сечений расслоения стационарных подалгебр над расширенным полем. Для этого необходимо решить систему уравнений на стабилизатор элемента общего положения.

Система на стабилизатор общего положения имеет следующий вид:

$$
\sum_{j=3}^{4} \sum_{p=1}^{2} x_{j} h_{p} a_{j i}^{p}=0, \quad i=1,2 .
$$


Напомним, что $h=\left(h_{1}, h_{2}\right)$ - элемент общего положения из пространства, двойственного к идеалу, а $x=\left(x_{1}, x_{2}, x_{3}, x_{4}\right)$ - элемент алгебры $A_{4,8}$. При фиксированном $h$ пространство решений системы и будет являться стабилизатором элемента $h$. В нашем случае система состоит из одного уравнения

$$
x_{3} h_{1}+x_{4} h_{2}=0 .
$$

Поэтому размерность стабилизатора элемента общего положения $\operatorname{dim} \operatorname{St}(h)=$ $4-1=3$, откуда получаем, что размерность алгебры рациональных сечений над расширенным полем $\operatorname{dim}_{\mathbb{R}\left(e_{1}, e_{2}\right)} L=3-2+1=2$. Следующие рациональные сечения образуют базис в алгебре рациональных сечений:

$$
\Psi_{0}:\left\{\begin{array}{l}
x_{1}=1, \\
x_{2}=0, \\
x_{3}=0, \\
x_{4}=0 ;
\end{array} \quad \Psi_{1}:\left\{\begin{array}{l}
x_{1}=0, \\
x_{2}=0, \\
x_{3}=1, \\
x_{4}=-\frac{h_{1}}{h_{2}} .
\end{array}\right.\right.
$$

Соответствующие им полиномы в алгебре $L$ имеют такой вид:

$$
f_{\Psi_{0}}=e_{1}, \quad f_{\Psi_{1}}=e_{2} e_{3}-e_{1} e_{4} .
$$

Выше мы установили, что алгебра $L$ двумерна, но в алгебре рациональных сечений расслоения стационарных подалгебр всегда существует центр, поэтому алгебра $L$ коммутативна. Следовательно, а значит в качестве полного коммутативного набора на ней можно взять просто линейные полиномы $f_{\Psi_{0}}$ и $f_{\Psi_{1}}$.

Чтобы получить полный коммутативный набор на первоначальной алгебpe $A_{4,8}$, необходимо к полиномам, соответствующим полному набору на алгебpe $L$, добавить базис в идеале (линейные полиномы). В итоге получаем, что $e_{1}, e_{2}, e_{2} e_{3}-e_{1} e_{4}-$ полный коммутативный набор на алгебре $A_{4,8}$.

Для остальных алгебр данного класса построение интегралов и соответствующие вычисления проводятся аналогично, и поэтому мы их опускаем. Полученные результаты приведены в таблицах 1-4.

Класс IV. Алгебры, в которых существует коммутативный идеал, а алгебра рациональных сечений трехмерна. Еще один класс состоит из четырех разрешимых алгебр $A_{4,11}, A_{5,25}, A_{5,26}$ и $A_{5,72}$. В этих алгебрах существует коммутативный идеал, не являющийся одномерным центром, такой, что алгебра рациональных сечений расслоения стационарных подалгебр над расширенным полем трехмерна.

УТВЕРЖДЕНИЕ 4. На всех алгебрах Ли класса IV явно указана интегрируемая гамильтонова система с полиномиальными первыми интегралами (см. табличьь 1-4).

Для построения на алгебре Ли интегрируемой гамильтоновой системы с полиномиальными первыми интегралами достаточно найти полный коммутативный набор полиномов на данной алгебре. Будем строить полный коммутативный набор полиномов по алгоритму Садэтова. Для алгебр класса IV алгоритм Садэтова будет состоять из следующих шагов: 
1) В первоначальной алгебре Ли есть коммутативный идеал, не являющийся одномерным центром, поэтому базисные элементы идеала образуют часть полного коммутативного набора. Для нахождения оставшейся части полного коммутативного набора необходимо рассмотреть алгебру рациональных сечений.

2) Алгебра рациональных сечений трехмерна. В ней, в свою очередь, существует коммутативный идеал, не являющийся одномерным центром. Добавляем базисные элементы данного идеала к уже построенной части полного коммутативного набора и переходим к следующей алгебре рациональных сечений.

3) Так как коммутативный идеал имеет большую размерность, то алгебра рациональных сечений одномерна и, следовательно, коммутативна. Поэтому полный коммутативный набор полиномов на ней строится тривиально.

4) Так как алгебра рациональных сечений рассматривалась над расширенным полем, то некоторые из полученных полиномов над первоначальным полем могут оказаться рациональными функциями. Умножая рациональные функции на соответствующие знаменатели, получаем полный коммутативный набор полиномов над исходным полем.

Рассмотрим алгебру $A_{5,25}$.

\begin{tabular}{|c|c|c|c|c|c|}
\hline \multirow{5}{*}{$A_{5,25}:$} & 0 & 0 & 0 & 0 & $2 p e_{1}$ \\
\hline & 0 & 0 & $e_{1}$ & 0 & $p e_{2}+e_{3}$ \\
\hline & 0 & $-e_{1}$ & 0 & 0 & $p e_{3}-e_{2}$ \\
\hline & 0 & 0 & 0 & 0 & $b e_{4}$ \\
\hline & $-2 p e_{1}$ & $-p e_{2}-e_{3}$ & $-p e_{3}+e_{2}$ & $-b e_{4}$ & 0 \\
\hline
\end{tabular}

Отметим, что это не одна алгебра, а целое семейство алгебр, зависящее от двух параметров $b$ и $p$, причем на параметры наложено единственное условие $b \neq 0$. Ее размерность $\operatorname{dim} A_{5,25}=5$, а индекс ind $A_{5,25}=1$. Поэтому полный коммутативный набор должен содержать $k=(5+1) / 2=3$ полинома. В этой алгебре существует коммутативный идеал, он натянут на векторы $e_{1}$ и $e_{4}$.

Теперь согласно методу Садэтова нужно перейти к алгебре рациональных сечений расслоения стационарных подалгебр над расширенным полем. Для этого необходимо решить систему на стабилизатор общего положения, которая в нашем случае будет иметь следующий вид:

$$
\left\{\begin{array}{l}
-2 p h_{1} x_{5}=0 \\
-b h_{4} x_{5}=0
\end{array}\right.
$$

Так как $h$ - элемент общего положения, то стабилизатор будет задаваться одним уравнением $x_{5}=0$. Поэтому размерность стабилизатора общего положения $\operatorname{dim} \operatorname{St}(h)=5-1=4$, а размерность алгебры рациональных сечений расслоения стационарных подалгебр над расширенным полем $\operatorname{dim}_{\mathbb{R}\left(e_{1}, e_{4}\right)} L=$ $4-2+1=3$. Следующие рациональные сечения образуют базис в алгебре 
рациональных сечений:

$$
\Psi_{0}:\left\{\begin{array}{l}
x_{1}=1, \\
x_{2}=0, \\
x_{3}=0, \\
x_{4}=0, \\
x_{5}=0 ;
\end{array} \quad \Psi_{1}:\left\{\begin{array}{l}
x_{1}=0, \\
x_{2}=1, \\
x_{3}=0, \\
x_{4}=0, \\
x_{5}=0 ;
\end{array} \quad \Psi_{2}:\left\{\begin{array}{l}
x_{1}=0, \\
x_{2}=0, \\
x_{3}=1, \\
x_{4}=0, \\
x_{5}=0 .
\end{array}\right.\right.\right.
$$

Соответствующие им полиномы в алгебре $L$ будут иметь следующий вид:

$$
f_{\Psi_{0}}=e_{1}, \quad f_{\Psi_{1}}=e_{2}, \quad f_{\Psi_{2}}=e_{3}
$$

Легко видеть, что таблица умножения алгебры $L$ в базисе $f_{\Psi_{0}}, f_{\Psi_{1}}, f_{\Psi_{2}}$ такова:

$L$ :

\begin{tabular}{|c|c|c|}
\hline 0 & 0 & 0 \\
\hline 0 & 0 & $f_{\Psi_{0}}$ \\
\hline 0 & $-f_{\Psi_{0}}$ & 0 \\
\hline
\end{tabular}

Подчеркнем, что это алгебра над полем $\mathbb{R}\left(e_{1}, e_{4}\right)$. Так как $\operatorname{dim} L=3$, а ind $L=1$, то полный коммутативный набор на алгебре $L$ должен состоять из $\widetilde{k}=(3+1) / 2=2$ полиномов. Но в алгебре $L$ существует большой коммутативный идеал, натянутый на векторы $f_{\Psi_{0}}$ и $f_{\Psi_{1}}$, поэтому в качестве полного коммутативного набора можно взять линейные полиномы (базисные векторы данного идеала).

Теперь добавим к полученным полиномам базисные векторы коммутативного идеала в алгебре $A_{5,25}$. Окончательно получаем, что $e_{1}, e_{2}, e_{4}$ - полный коммутативный набор полиномов на исходной алгебре $A_{5,25}$.

Для остальных алгебр данного класса построения интегралов и соответствующие вычисления проводятся аналогично, и поэтому мы их опускаем. Полученные результаты приведены в таблицах 1-4.

Класс V. Алгебра, в которой существует идеал, изоморфный алгебре Гейзенберга, а подалгебра $\mathfrak{b}$ двумерна. В этом пункте мы разберем класс, состоящий из одной разрешимой алгебры - $A_{4,10}$.

УТВЕРЖДЕНИЕ 5. На единственной алгебре Ли, образующей класс $\mathrm{V}$, явно указана интегрируемая гамильтонова система с полиномиальными первыми интегралами (см. таблищъ 1-4).

Рассмотрим алгебру $A_{4,10}$.

$A_{4,10}:$\begin{tabular}{|c|c|c|c|}
\hline 0 & 0 & 0 & 0 \\
\hline 0 & 0 & $e_{1}$ & $-e_{3}$ \\
\hline 0 & $-e_{1}$ & 0 & $e_{2}$ \\
\hline 0 & $e_{3}$ & $-e_{2}$ & 0 \\
\hline
\end{tabular}

Ее размерность $\operatorname{dim} A_{4,10}=4$, а индекс ind $A_{4,10}=2$, поэтому полный коммутативный набор должен состоять из $k=(4+2) / 2=3$ полиномов. В этой 
алгебре не существует коммутативного идеала, не совпадающего с одномерным центром алгебры, но есть идеал, изоморфный алгебре Гейзенберга $\mathfrak{h}_{1}$, причем центр идеала совпадает с центром алгебры. Идеал натянут на базисные векторы $e_{1}, e_{2}, e_{3}$, где $e_{1}$ является центром как идеала, так и алгебры $A_{4,10}$.

Согласно методу Садэтова теперь необходимо рассмотреть проекцию алгебры $A_{4,10}$ на некоторую подалгебру $\mathfrak{b}$. В данном случае подалгебра $\mathfrak{b}$ натянута на $e_{1}$ и $e_{4}$. Для данной алгебры $\mathfrak{b}$ существует вложение в $P\left(A_{4,10}\right)$, которое устроено следующим образом:

$$
f_{\beta}(x)=\langle\beta, x\rangle\left\langle e_{1}, x\right\rangle+\frac{1}{2}\left\langle\omega^{-1}\left(\left(\operatorname{ad}_{\beta}\right)^{*} \operatorname{pr}_{V}(x)\right), x\right\rangle
$$

где $\beta \in \mathfrak{b}$ - произвольный элемент алгебры $\mathfrak{b}, f_{\beta}$ - образ вложения (квадратичный полином на $\left.A_{4,10}^{*}\right), x$ - произвольный элемент из $A_{4,10}^{*}$

Далее необходимо построить полный коммутативный набор полиномов на алгебре $\mathfrak{b}$ и взять образ полного коммутативного набора при описанном выше вложении. $\mathrm{K}$ полученным полиномам добавить полный набор на $\mathfrak{h}_{1}$.

В нашем случае подалгебра $\mathfrak{b}$ двумерна и коммутативна, поэтому в качестве полного коммутативного набора на ней достаточно взять базисные элементы (линейные полиномы), т.е. $e_{1}$ и $e_{4}$. Легко проверить, что их образами при вложении будут следующие полиномы:

$$
f_{e_{1}}=e_{1}^{2}, \quad f_{e_{4}}=e_{1} e_{4}+\frac{1}{2}\left(e_{2}^{2}+e_{3}^{2}\right)
$$

Добавляя к ним $e_{1}, e_{2}-$ полный коммутативный набор на $\mathfrak{h}_{1}$, получаем, что полиномы $e_{1}, e_{2}, e_{1} e_{4}+\left(e_{2}^{2}+e_{3}^{2}\right) / 2$ образуют полный коммутативный набор на исходной алгебре $A_{4,10}$.

Класс VI. Алгебры, в которых существует коммутативный идеал, не совпадающий с одномерным центром, а алгебра рациональных сечений содержит идеал, изоморфный алгебре Гейзенберга. Этот класс состоит из двух разрешимых алгебр: $A_{5,37}$ и $A_{5,71}$.

УТВЕРЖДЕНИЕ 6. На всех алгебрах Ли класса VI явно указана интегрируемая гамильтонова система с полиномиальными первыми интегралами (см. таблииљ 1-4).

Для построения на алгебре Ли интегрируемой гамильтоновой системы с полиномиальными первыми интегралами достаточно найти полный коммутативный набор полиномов на данной алгебре. Будем строить полный коммутативный набор полиномов по алгоритму Садэтова. Для алгебр класса VI алгоритм Садэтова будет состоять из следующих шагов:

1) В первоначальной алгебре Ли есть коммутативный идеал, не являющийся одномерным центром, поэтому базисные элементы идеала образуют часть полного коммутативного набора. Для нахождения оставшейся части полного коммутативного набора необходимо рассмотреть алгебру рациональных сечений. 
2) Алгебра рациональных сечений содержит идеал, изоморфный алгебре Гейзенберга, причем центр идеала совпадает с одномерным центром всей алгебры. Поэтому необходимо рассмотреть проекцию алгебры рациональных сечений на подалгебру $\mathfrak{b}$. Построение оставшейся части полного коммутативного набора сводится к построению полного коммутативного набора на подалгебре $\mathfrak{b}$.

3) Подалгебра $\mathfrak{b}$ коммутативна, полный коммутативный набор полиномов на ней строится тривиально.

Рассмотрим алгебру Ли $A_{5,37}$.

$A_{5,37}:$\begin{tabular}{|c|c|c|c|c|}
\hline 0 & 0 & 0 & $2 e_{1}$ & 0 \\
\hline 0 & 0 & $e_{1}$ & $e_{2}$ & $-e_{3}$ \\
\hline 0 & $-e_{1}$ & 0 & $e_{3}$ & $e_{2}$ \\
\hline$-2 e_{1}$ & $-e_{2}$ & $-e_{3}$ & 0 & 0 \\
\hline 0 & $e_{3}$ & $-e_{2}$ & 0 & 0 \\
\hline
\end{tabular}

Размерность этой алгебры $\operatorname{dim} A_{5,37}=5$, а индекс ind $A_{5,37}=1$, поэтому полный коммутативный набор должен состоять из $k=(5+1) / 2=3$ полиномов.

В этой алгебре существует одномерный коммутативный идеал, не являющийся одномерным центром. Он натянут на вектор $e_{1}$. Переходя к алгебре рациональных сечений расслоения стационарных подалгебр, находим стабилизатор общего положения, который задается системой, состоящей из одного уравнения

$$
-2 h_{1} x_{4}=0
$$

Так как $h$ - элемент общего положения, то стабилизатор общего положения задается уравнением $x_{4}=0$. Поэтому $\operatorname{dim} \operatorname{St}(h)=5-1=4, \operatorname{adim}_{\mathbb{R}\left(e_{1}\right)} L=$ $4-1+1=4$. Следующие рациональные сечения образуют базис в алгебре рациональных сечений:

$$
\Psi_{0}:\left\{\begin{array}{l}
x_{1}=1, \\
x_{2}=0, \\
x_{3}=0, \\
x_{4}=0, \\
x_{5}=0 ;
\end{array} \quad \Psi_{1}:\left\{\begin{array}{l}
x_{1}=0, \\
x_{2}=1, \\
x_{3}=0, \\
x_{4}=0, \\
x_{5}=0 ;
\end{array} \quad \Psi_{2}:\left\{\begin{array}{l}
x_{1}=0, \\
x_{2}=0, \\
x_{3}=1, \\
x_{4}=0, \\
x_{5}=0 ;
\end{array} \quad \Psi_{3}:\left\{\begin{array}{l}
x_{1}=0, \\
x_{2}=0, \\
x_{3}=0, \\
x_{4}=0, \\
x_{5}=1 .
\end{array}\right.\right.\right.\right.
$$

Соответствующие им полиномы в алгебре $L$ имеют следующий вид:

$$
f_{\Psi_{0}}=e_{1}, \quad f_{\Psi_{1}}=e_{2}, \quad f_{\Psi_{2}}=e_{3}, \quad f_{\Psi_{3}}=e_{5} .
$$

Поэтому таблица умножения алгебры $L$ в базисе $f_{\Psi_{0}}, f_{\Psi_{1}}, f_{\Psi_{2}}, f_{\Psi_{3}}$ такова:

$L:$\begin{tabular}{|c|c|c|c|}
\hline 0 & 0 & 0 & 0 \\
\hline 0 & 0 & $f_{\Psi_{0}}$ & $-f_{\Psi_{2}}$ \\
\hline 0 & $-f_{\Psi_{0}}$ & 0 & $f_{\Psi_{1}}$ \\
\hline 0 & $f_{\Psi_{2}}$ & $-f_{\Psi_{1}}$ & 0 \\
\hline
\end{tabular}


Taк как $\operatorname{ind} L=2$, a $\operatorname{dim} L=4$, то полный коммутативный набор должен состоять из $\widetilde{k}=(4+2) / 2=3$ полиномов. Несмотря на то, что алгебра $L$ над полем $\mathbb{R}\left(e_{1}\right)$, ее структурные константы в данном базисе принадлежат $\mathbb{R}$ и совпадают со структурными константами алгебры $A_{4,10}$. Поэтому в качестве полного коммутативного набора на алгебре $L$ можно взять полный коммутативный набор на алгебре $A_{4,10}$. Добавляя к нему базисный вектор одномерного идеала (линейный полином), получаем, что $e_{1}, e_{2}, e_{1} e_{5}+\left(e_{2}^{2}+e_{3}^{2}\right) / 2$ образуют полный коммутативный набор полиномов на исходной алгебре $A_{5,37}$.

Для второй алгебры данного класса построение интегралов и соответствующие вычисления проводятся аналогично, и поэтому мы их опускаем. Полученные результаты приведены в таблицах 1-4.

Класс VII. Алгебры, в которых существует одномерный коммутативный идеал, не совпадающий с одномерным центром, а алгебра рациональных сечений редуктивна. Мы переходим к рассмотрению последнего, седьмого класса, состоящего из двух разложимых алгебр общего типа $A_{5,60}, A_{5,61}$.

УТВЕРЖДЕНИЕ 7. На всех алгебрах Ли класса VII существует интегрируемая гамильтонова система с полиномиальными первыми интегралами (см. таблицы 1-4).

Для построения на алгебре Ли интегрируемой гамильтоновой системы с полиномиальными первыми интегралами достаточно найти полный коммутативный набор полиномов на данной алгебре. Будем строить полный коммутативный набор полиномов по алгоритму Садэтова. Для алгебр класса VII этот алгоритм будет состоять из следующих шагов.

1) В первоначальной алгебре Ли есть одномерный коммутативный идеал, не являющийся одномерным центром, поэтому базисный элемент идеала образует часть полного коммутативного набора. Для нахождения оставшейся части полного коммутативного набора необходимо рассмотреть алгебру рациональных сечений.

2) Алгебра рациональных сечений редуктивна, поэтому построение на ней полного коммутативного набора полиномов производится методом сдвига аргумента.

Рассмотрим алгебру $A_{5,60}$.

$A_{5,60}:$\begin{tabular}{|c|c|c|c|c|}
\hline 0 & $e_{1}$ & $-2 e_{2}$ & 0 & 0 \\
\hline$-e_{1}$ & 0 & $e_{3}$ & 0 & 0 \\
\hline $2 e_{2}$ & $-e_{3}$ & 0 & 0 & 0 \\
\hline 0 & 0 & 0 & 0 & $e_{4}$ \\
\hline 0 & 0 & 0 & $-e_{4}$ & 0 \\
\hline
\end{tabular}

Так как $\operatorname{dim} A_{5,60}=5$, a ind $A_{5,60}=1$, то полный коммутативный набор должен состоять из $k=(5+1) / 2=3$ полиномов.

В этой алгебре существует одномерный коммутативный идеал, не являющийся одномерным центром. Он натянут на вектор $e_{4}$. Переходя к алгебре 
рациональных сечений расслоения стационарных подалгебр, находим стабилизатор общего положения, который задается системой, состоящей из одного уравнения

$$
h_{4} x_{5}=0
$$

Так как $h$ - элемент общего положения, то стабилизатор общего положения задается уравнением: $x_{5}=0$. Поэтому $\operatorname{dim} \operatorname{St}(h)=5-1=4, \operatorname{dim}_{\mathbb{R}\left(e_{4}\right)} L=$ $4-1+1=4$. Следующие рациональные сечения образуют базис в алгебре рациональных сечений:

$$
\Psi_{0}:\left\{\begin{array}{l}
x_{1}=1, \\
x_{2}=0, \\
x_{3}=0, \\
x_{4}=0, \\
x_{5}=0 ;
\end{array} \quad \Psi_{1}:\left\{\begin{array}{l}
x_{1}=0, \\
x_{2}=1, \\
x_{3}=0, \\
x_{4}=0, \\
x_{5}=0 ;
\end{array} \quad \Psi_{2}:\left\{\begin{array}{l}
x_{1}=0, \\
x_{2}=0, \\
x_{3}=1, \\
x_{4}=0, \\
x_{5}=0 ;
\end{array} \quad \Psi_{3}:\left\{\begin{array}{l}
x_{1}=0, \\
x_{2}=0, \\
x_{3}=0, \\
x_{4}=1, \\
x_{5}=0 .
\end{array}\right.\right.\right.\right.
$$

Следующие полиномы в $L$ соответствуют данным базисным рациональным сечениям:

$$
f_{\Psi_{0}}=e_{1}, \quad f_{\Psi_{1}}=e_{2}, \quad f_{\Psi_{2}}=e_{3}, \quad f_{\Psi_{3}}=e_{4}
$$

Поэтому таблица умножения алгебры $L$ в базисе $f_{\Psi_{0}}, f_{\Psi_{1}}, f_{\Psi_{2}}, f_{\Psi_{3}}$ будет выглядеть так:

$L:$\begin{tabular}{|c|c|c|c|}
\hline 0 & $f_{\Psi_{0}}$ & $-2 f_{\Psi_{1}}$ & 0 \\
\hline$-f_{\Psi_{0}}$ & 0 & $f_{\Psi_{2}}$ & 0 \\
\hline $2 f_{\Psi_{1}}$ & $-f_{\Psi_{2}}$ & 0 & 0 \\
\hline 0 & 0 & 0 & 0 \\
\hline
\end{tabular}

Алгебра $L$ над полем $\mathbb{R}\left(e_{4}\right)$ редуктивна. Поэтому, следуя алгоритму Садэтова, полный коммутативный набор на ней находится методом сдвига аргумента. Этот метод изложен в работах Мищенко, Фоменко [5], [6] и здесь мы опустим описание его применения.

Для второй алгебры данного класса построение интегралов и соответствующие вычисления проводятся аналогично, и поэтому мы их опускаем. Полученные результаты приведены в таблицах 1-4.

Описанные выше построения обобщает следующая теорема.

ТЕОрема 4. На вещественных алгебрах Ли малой размерности построень следующие интегрируемые гамильтоновы системы с полиномиальными первыми интегралами.

1) Случай трехмерных вещественных алгебр Ли.

1.1) На каждой неразложимой трехмерной вещественной неполупростой алгебре Ли (в том числе и на двух семействах алгебр, зависящих от континуального параметра) явно указана интегрируемая гамильтонова система с линейными первыми интегралами (см. таблииу 1). 
1.2) На каждой разложимой трехмерной вещественной алгебре Ли явно указана интегрируемая гамильтонова система с линейными первыми интегралами (см. табличу 1).

2) Случай четырехмерных вещественных алгебр Ли.

2.1) На неразложимой алгебре $A_{4,8}$ с коммутатором

$$
\left[e_{2}, e_{3}\right]=e_{1}, \quad\left[e_{2}, e_{4}\right]=e_{2}, \quad\left[e_{3}, e_{4}\right]=-e_{3}
$$

найдена интегрируемая гамильтонова система со следующими линейными и квадратичным первыми интегралами: $y_{1}, y_{2}, y_{2} y_{3}-y_{1} y_{4}$.

2.2) На неразложимой алгебре $A_{4,10}$ с коммутатором

$$
\left[e_{2}, e_{3}\right]=e_{1}, \quad\left[e_{2}, e_{4}\right]=-e_{3}, \quad\left[e_{3}, e_{4}\right]=e_{2}
$$

найдена интегрируемая гамильтонова система со следующими линейными и квадратичным первыми интегралами: $y_{1}, y_{2}, y_{1} y_{4}+\left(y_{2}^{2}+y_{3}^{2}\right) / 2$.

2.3) На всех остальных неразложимых четырехмерных вещественных алгебрах Ли (в том числе и на пяти семействах алгебр, зависящих от континуального параметра) явно указана интегрируемая гамильтонова система с линейными первыми интегралами (см. таблицу 2).

2.4) На всех разложимых нередуктивных четырехмерных вещественных алгебрах Ли (в том числе и на двух семействах алгебр, зависящих от континуального параметра) явно указана интегрируемая гамильтонова система с линейными первыми интегралами (см. таблицу 2).

3) Случай пятимерных вещественных алгебр Ли.

3.1) На неразложимой алгебре $A_{5,3}$ с коммутатором

$$
\left[e_{3}, e_{4}\right]=e_{2}, \quad\left[e_{3}, e_{5}\right]=e_{1}, \quad\left[e_{4}, e_{5}\right]=e_{3}
$$

найдена интегрируемая гамильтонова система со следующими линейными и квадратичным первыми интегралами: $y_{1}, y_{2}, y_{3}, y_{1} y_{4}-y_{2} y_{5}$.

3.2) На неразложимой алгебре $A_{5,36}$ с коммутатором

$$
\begin{gathered}
{\left[e_{2}, e_{3}\right]=e_{1}, \quad\left[e_{1}, e_{4}\right]=e_{1}, \quad\left[e_{2}, e_{4}\right]=e_{2},} \\
{\left[e_{2}, e_{5}\right]=-e_{2}, \quad\left[e_{3}, e_{5}\right]=e_{3}}
\end{gathered}
$$

найдена интегрируемая гамильтонова система со следующими линейными и квадратичным первыми интегралами: $y_{1}, y_{2}, y_{2} y_{3}+y_{1} y_{5}$.

3.3) На неразложимой алгебре $A_{5,37}$ с коммутатором

$$
\begin{array}{llrl}
{\left[e_{2}, e_{3}\right]} & =e_{1}, & {\left[e_{1}, e_{4}\right]=2 e_{1},} & {\left[e_{2}, e_{4}\right]=e_{2},} \\
{\left[e_{3}, e_{4}\right]=e_{3},} & {\left[e_{2}, e_{5}\right]=-e_{3},} & {\left[e_{3}, e_{5}\right]=e_{2}}
\end{array}
$$

найдена интегрируемая гамильтонова система со следующими линейными и квадратичным первыми интегралами: $y_{1}, y_{2}, y_{1} y_{5}+\left(y_{2}^{2}+y_{3}^{2}\right) / 2$. 
3.4) На неразложимой алгебре $A_{5,40}$ с коммутатором

$$
\begin{gathered}
{\left[e_{1}, e_{2}\right]=2 e_{1}, \quad\left[e_{1}, e_{3}\right]=-e_{2}, \quad\left[e_{2}, e_{3}\right]=2 e_{3}, \quad\left[e_{1}, e_{4}\right]=e_{5}} \\
{\left[e_{2}, e_{4}\right]=e_{4}, \quad\left[e_{2}, e_{5}\right]=-e_{5}, \quad\left[e_{3}, e_{5}\right]=e_{4}}
\end{gathered}
$$

найдена интегрируемая гамильтонова система со следующими линейными и кубическим первыми интегралами: $y_{2} y_{4} y_{5}-y_{1} y_{4}^{2}+y_{3} y_{5}^{2}, y_{4}, y_{5}$.

3.5) На всех остальных неразложимых пятимерных вещественных алгебрах Ли (в том числе и на двадиати одном семействе алгебр, зависящих от континуального параметра) явно указана интегрируемая гамильтонова система с линейными первыми интегралами (см. таблицу 3).

3.6) На разложимой алгебре $A_{5,60}$ с коммутатором

$$
\left[e_{1}, e_{3}\right]=-2 e_{2}, \quad\left[e_{1}, e_{2}\right]=e_{1}, \quad\left[e_{2}, e_{3}\right]=e_{3}, \quad\left[e_{4}, e_{5}\right]=e_{4}
$$

существует интегрируемая гамильтонова система с линейными и квадратичным первыми интегралами.

3.7) На разложимой алгебре $A_{5,61}$ с коммутатором

$$
\left[e_{1}, e_{2}\right]=e_{3}, \quad\left[e_{2}, e_{3}\right]=e_{1}, \quad\left[e_{3}, e_{1}\right]=e_{2}, \quad\left[e_{4}, e_{5}\right]=e_{4}
$$

существует интегрируемая гамильтонова система с линейными и квадратичным первыми интегралами.

3.8) На разложимой алгебре $A_{5,69}$ с коммутатором

$$
\left[e_{2}, e_{3}\right]=e_{1}, \quad\left[e_{2}, e_{4}\right]=e_{2}, \quad\left[e_{3}, e_{4}\right]=-e_{3}
$$

найдена интегрируемая гамильтонова система со следующими линейными и квадратичным первыми интегралами: $y_{1}, y_{2}, y_{5}, y_{2} y_{3}-y_{1} y_{4}$.

3.9) На разложимой алгебре $A_{5,71}$ с коммутатором

$$
\left[e_{2}, e_{3}\right]=e_{1}, \quad\left[e_{2}, e_{4}\right]=-e_{3}, \quad\left[e_{3}, e_{4}\right]=e_{2}
$$

найдена интегрируемая гамильтонова система со следующими линейными и квадратичным первыми интегралами: $y_{1}, y_{2}, y_{5}, y_{1} y_{4}+\frac{1}{2}\left(y_{2}^{2}+y_{3}^{2}\right)$.

3.10) На всех остальных разложимых нередуктивных пятимерных вещественных алгебрах Ли (в том числе и на девяти семействах алгебр, зависящих от континуального параметра) явно указана интегрируемая гамильтонова система с линейными первыми интегралами (см. табличу 3).

4) Случай шестимерных нильпотентных алгебр Ли.

4.1) На неразложимой алгебре $A_{6,3}$ с коммутатором

$$
\left[e_{1}, e_{2}\right]=e_{6}, \quad\left[e_{1}, e_{3}\right]=e_{4}, \quad\left[e_{2}, e_{3}\right]=e_{5}
$$

найдена интегрируемая гамильтонова система со следующими линейными и квадратичным первыми интегралами: $y_{3}, y_{4}, y_{5}, y_{6}, y_{2} y_{4}-y_{1} y_{5}$. 
4.2) На неразложимой алгебре $A_{6,21}$ с коммутатором

$$
\begin{gathered}
{\left[e_{1}, e_{2}\right]=e_{3}, \quad\left[e_{1}, e_{5}\right]=e_{6}, \quad\left[e_{2}, e_{3}\right]=e_{4},} \\
{\left[e_{2}, e_{4}\right]=e_{5}, \quad\left[e_{3}, e_{4}\right]=e_{6}}
\end{gathered}
$$

найдена интегрируемая гамильтонова система со следующими линейными и квадратичным первыми интегралами: $y_{4}, y_{5}, y_{6}, y_{2} y_{6}-y_{3} y_{5}$.

4.3) На неразложимой алгебре $A_{6,22}$ с коммутатором

$$
\begin{array}{lll}
{\left[e_{1}, e_{2}\right]=e_{3},} & {\left[e_{1}, e_{3}\right]=e_{5},} & {\left[e_{1}, e_{5}\right]=e_{6},} \\
{\left[e_{2}, e_{3}\right]=e_{4},} & {\left[e_{2}, e_{4}\right]=e_{5},} & {\left[e_{3}, e_{4}\right]=e_{6}}
\end{array}
$$

найдена интегрируемая гамильтонова система со следующими линейными и квадратичным первыми интегралами: $y_{4}, y_{5}, y_{6}, y_{2} y_{6}-y_{3} y_{5}$.

4.4) На всех остальных неразложимых шестимерных вещественных нильпотентных алгебрах Ли (в том числе и на двух семействах алгебр, зависящих от континуального параметра) явно указана интегрируемая гамильтонова система с линейными первыми интегралами (см. таблииу 4).

4.5) На разложимой алгебре $A_{6,29}$ с коммутатором

$$
\left[e_{3}, e_{4}\right]=e_{2}, \quad\left[e_{3}, e_{5}\right]=e_{1}, \quad\left[e_{4}, e_{5}\right]=e_{3}
$$

найдена интегрируемая гамильтонова система со следующими линейными и квадратичным первыми интегралами: $y_{1}, y_{2}, y_{3}, y_{6}, y_{1} y_{4}-y_{2} y_{5}$.

4.6) На всех остальных разложимых шестимерных вещественных нильпотентных алгебрах Ли явно указана интегрируемая гамильтонова система с линейными первыми интегралами (см. табличу 4).

Доказательство этой теоремы следует из утверждений 1-7.

Из нее следует, что среди алгебр Ли малой размерности существуют две исключительные трехмерные алгебры, две четырехмерные, семь пятимерных, четыре шестимерных нильпотентных, на которых полный коммутативный набор состоит из линейных и квадратичных полиномов. Существует одна исключительная пятимерная алгебра Ли, на которой полный коммутативный набор состоит из кубического и линейных полиномов, причем данная алгебра является единственной неразложимой алгеброй общего типа из рассмотренных. На остальных алгебрах Ли малой размерности существует полный коммутативный набор линейных полиномов.

Также имеет место следующий интересный эффект: некоторые неизоморфные алгебры образуют семейства, зависящие от континуального параметра, однако полные коммутативные наборы полиномов на них совпадают и не зависят от параметра.

Известна гипотеза, выдвинутая М.В. Миловановым относительно полных коммутативных наборов полиномов на разрешимых алгебрах Ли.

ГипотезА 2 (М.В. Милованов). На любой разрешимой алгебре Ли существует полный коммутативный набор из линейных и квадратичных полиномов. 
На всех рассмотренных разрешимых алгебрах Ли полные коммутативные наборы состоят из линейных и квадратичных полиномов. Поэтому контрпример к этой гипотезе может существовать только для алгебр Ли высоких размерностей.

ТАБлицА 1. Все трехмерные вещественные алгебры Ли

\begin{tabular}{|c|c|c|c|c|}
\hline \multicolumn{5}{|c|}{ Неразложсимые } \\
\hline $\begin{array}{l}A_{3,1} \\
\text { Класс II }\end{array}$ & {$\left[e_{2}, e_{3}\right]=e_{1}$} & Нильпотентная & $x_{1}$ & $y_{1}, y_{2}$ \\
\hline $\begin{array}{l}A_{3,2} \\
\text { Класс II }\end{array}$ & $\begin{array}{l}{\left[e_{1}, e_{3}\right]=e_{1}} \\
{\left[e_{2}, e_{3}\right]=e_{1}+e_{2}}\end{array}$ & Разрешимая & $x_{1} \exp \left[-\frac{x_{2}}{x_{1}}\right]$ & $y_{1}, y_{2}$ \\
\hline $\begin{array}{l}A_{3,3} \\
\text { Класс II }\end{array}$ & $\begin{array}{l}{\left[e_{1}, e_{3}\right]=e_{1}} \\
{\left[e_{2}, e_{3}\right]=e_{2}}\end{array}$ & Разрешимая & $\frac{x_{2}}{x_{1}}$ & $y_{1}, y_{2}$ \\
\hline $\begin{array}{l}A_{3,4} \\
\text { Класс II }\end{array}$ & $\begin{array}{l}{\left[e_{1}, e_{3}\right]=e_{1},} \\
{\left[e_{2}, e_{3}\right]=-e_{2}}\end{array}$ & Разрешимая & $x_{1} x_{2}$ & $y_{1}, y_{2}$ \\
\hline $\begin{array}{l}A_{3,5} \\
\text { Класс II }\end{array}$ & $\begin{array}{l}{\left[e_{1}, e_{3}\right]=e_{1},} \\
{\left[e_{2}, e_{3}\right]=a e_{2}}\end{array}$ & Разрешимая & $0<|a|<1, x_{2} x_{1}^{-a}$ & $y_{1}, y_{2}$ \\
\hline $\begin{array}{l}A_{3,6} \\
\text { Класс II }\end{array}$ & $\begin{array}{l}{\left[e_{1}, e_{3}\right]=-e_{2}} \\
{\left[e_{2}, e_{3}\right]=e_{1}}\end{array}$ & Разрешимая & $x_{1}^{2}+x_{2}^{2}$ & $y_{1}, y_{2}$ \\
\hline $\begin{array}{l}A_{3,7} \\
\text { Класс II }\end{array}$ & $\begin{array}{l}{\left[e_{1}, e_{3}\right]=a e_{1}-e_{2},} \\
{\left[e_{2}, e_{3}\right]=e_{1}+a e_{2}}\end{array}$ & Разрешимая & $\begin{array}{l}a>0, \\
\left(x_{1}^{2}+x_{2}^{2}\right)\left[\frac{x_{1}+i x_{2}}{x_{1}-i x_{2}}\right]^{i a}\end{array}$ & $y_{1}, y_{2}$ \\
\hline $\begin{array}{l}A_{3,8} \\
\text { Класс I }\end{array}$ & $\begin{array}{l}{\left[e_{1}, e_{3}\right]=-2 e_{2}} \\
{\left[e_{1}, e_{2}\right]=e_{1}} \\
{\left[e_{2}, e_{3}\right]=e_{3}}\end{array}$ & Полупростая & $x_{2}^{2}+x_{1} x_{3}$ & $\begin{array}{l}\text { метод } \\
\text { сдвига } \\
\text { аргумента }\end{array}$ \\
\hline $\begin{array}{l}A_{3,9} \\
\text { Класс I }\end{array}$ & $\begin{array}{l}{\left[e_{1}, e_{2}\right]=e_{3}} \\
{\left[e_{2}, e_{3}\right]=e_{1}} \\
{\left[e_{3}, e_{1}\right]=e_{2}}\end{array}$ & Полупростая & $x_{1}^{2}+x_{2}^{2}+x_{3}^{2}$ & $\begin{array}{l}\text { метод } \\
\text { сдвига } \\
\text { аргумента } \\
\end{array}$ \\
\hline \multicolumn{5}{|c|}{ Разложимые } \\
\hline $\begin{array}{l}A_{3,10} \\
\text { Класс II }\end{array}$ & - & Абелева & $x_{1}, x_{2}, x_{3}$ & $y_{1}, y_{2}, y_{3}$ \\
\hline $\begin{array}{l}A_{3,11} \\
\text { Класс II }\end{array}$ & {$\left[e_{1}, e_{2}\right]=e_{1}$} & Разрешимая & $x_{3}$ & $y_{2}, y_{3}$ \\
\hline
\end{tabular}

ТАБлицА 2. Все четырехмерные вещественные алгебры Ли

\begin{tabular}{|l|l|l|l|l|}
\hline \multicolumn{5}{|c|}{ Неразложимье } \\
\hline $\begin{array}{l}A_{4,1} \\
\text { Класс II }\end{array}$ & $\begin{array}{l}{\left[e_{2}, e_{4}\right]=e_{1},} \\
{\left[e_{3}, e_{4}\right]=e_{2}}\end{array}$ & Нильпотентная & $x_{1}, x_{2}^{2}-2 x_{1} x_{3}$ & $y_{1}, y_{2}, y_{3}$ \\
\hline $\begin{array}{l}A_{4,2} \\
\text { Класс II }\end{array}$ & $\begin{array}{l}{\left[e_{1}, e_{4}\right]=e_{1},} \\
{\left[e_{2}, e_{4}\right]=e_{2},} \\
{\left[e_{3}, e_{4}\right]=e_{2}+e_{3}}\end{array}$ & Разрешимая & $a \neq 0, x_{2} \exp \left(-\frac{x_{3}}{x_{2}}\right), \frac{x_{2}^{a}}{x_{1}}$ & $y_{1}, y_{2}, y_{3}$ \\
\hline $\begin{array}{l}A_{4,3} \\
\text { Класс II }\end{array}$ & {$\left[e_{1}, e_{4}\right]=e_{1}$,} & Разрешимая & $x_{2}, x_{1} \exp \left(-\frac{x_{3}}{x_{2}}\right)$ & $y_{1}, y_{2}, y_{3}$ \\
\hline $\begin{array}{l}\left.A_{4,4}, e_{4}\right]=e_{2} \\
\text { Класс II }\end{array}$ & {$\left[e_{1}, e_{4}\right]=e_{1}$,} \\
{$\left[e_{2}, e_{4}\right]=e_{1}+e_{2}$,} & Разрешимая & $x_{1} \exp \left(-\frac{x_{2}}{x_{1}}\right), \frac{2 x_{1} x_{3}-x_{2}^{2}}{x_{1}^{2}}$ & $y_{1}, y_{2}, y_{3}$ \\
\hline
\end{tabular}


Таблица 2 (продолжение)

\begin{tabular}{|c|c|c|c|c|c|}
\hline $\begin{array}{l}A_{4,5} \\
\text { Класс II }\end{array}$ & $\begin{array}{l}{\left[e_{1}, e_{4}\right]} \\
{\left[e_{2}, e_{4}\right]} \\
{\left[e_{3}, e_{4}\right]}\end{array}$ & $\begin{array}{l}=e_{1} \\
=a e_{2} \\
=b e_{3}\end{array}$ & Разрешимая & $\begin{array}{l}a b \neq 0,-1 \leqslant b \leqslant a \leqslant 1, \\
\frac{x_{1}^{a}}{x_{2}}, \frac{x_{1}^{b}}{x_{3}}\end{array}$ & $y_{1}, y_{2}, y_{3}$ \\
\hline $\begin{array}{l}A_{4,6} \\
\text { Класс II }\end{array}$ & $\begin{array}{l}{\left[e_{1}, e_{4}\right]} \\
{\left[e_{2}, e_{4}\right]} \\
{\left[e_{3}, e_{4}\right]}\end{array}$ & $\begin{array}{l}=a e_{1} \\
=b e_{2}-e_{3} \\
=e_{2}+b e_{3}\end{array}$ & Разрешимая & $\begin{array}{l}a \neq 0, b \geqslant 0, \frac{x_{1}^{2 b / a}}{x_{2}^{2}+x_{3}^{2}} \\
\left(x_{2}^{2}+x_{3}^{2}\right)\left(\frac{x_{2}+i x_{3}}{x_{2}-i x_{3}}\right)^{i b}\end{array}$ & $y_{1}, y_{2}, y_{3}$ \\
\hline $\begin{array}{l}A_{4,7} \\
\text { Класс II }\end{array}$ & $\begin{array}{l}{\left[e_{2}, e_{3}\right]} \\
{\left[e_{1}, e_{4}\right]} \\
{\left[e_{2}, e_{4}\right]} \\
{\left[e_{3}, e_{4}\right]}\end{array}$ & $\begin{array}{l}=e_{1} \\
=2 e_{1} \\
=e_{2} \\
=e_{2}+e_{3}\end{array}$ & Разрешимая & нет & $y_{1}, y_{2}$ \\
\hline $\begin{array}{l}A_{4,8} \\
\text { Класс III }\end{array}$ & $\begin{array}{l}{\left[e_{2}, e_{3}\right]} \\
{\left[e_{2}, e_{4}\right]} \\
{\left[e_{3}, e_{4}\right]}\end{array}$ & $\begin{array}{l}=e_{1} \\
=e_{2} \\
=-e_{3}\end{array}$ & Разрешимая & $x_{1}, x_{2} x_{3}-x_{1} x_{4}$ & $\begin{array}{l}y_{1}, y_{2} \\
y_{2} y_{3}-y_{1} y_{4}\end{array}$ \\
\hline $\begin{array}{l}A_{4,9} \\
\text { Класс II }\end{array}$ & $\begin{array}{l}{\left[e_{2}, e_{3}\right]} \\
{\left[e_{1}, e_{4}\right]} \\
{\left[e_{2}, e_{4}\right]} \\
{\left[e_{3}, e_{4}\right]}\end{array}$ & $\begin{array}{l}=e_{1}, \\
=(1+b) e_{1}, \\
=e_{2}, \\
=b e_{3}\end{array}$ & Разрешимая & $\begin{array}{l}-1<b \leqslant 1 \\
\text { нет }\end{array}$ & $y_{1}, y_{2}$ \\
\hline $\begin{array}{l}A_{4,10} \\
\text { Класс V }\end{array}$ & $\begin{array}{l}{\left[e_{2}, e_{3}\right]} \\
{\left[e_{2}, e_{4}\right]} \\
{\left[e_{3}, e_{4}\right]}\end{array}$ & $\begin{array}{l}=e_{1} \\
=-e_{3} \\
=e_{2}\end{array}$ & Разрешимая & $x_{1}, 2 x_{1} x_{4}+x_{2}^{2}+x_{3}^{2}$ & $\begin{array}{l}y_{1}, y_{2} \\
y_{1} y_{4} \\
+\frac{1}{2}\left(y_{2}^{2}+y_{3}^{2}\right)\end{array}$ \\
\hline $\begin{array}{l}A_{4,11} \\
\text { Класс IV }\end{array}$ & $\begin{array}{l}{\left[e_{2}, e_{3}\right]} \\
{\left[e_{1}, e_{4}\right]} \\
{\left[e_{2}, e_{4}\right]} \\
{\left[e_{3}, e_{4}\right]}\end{array}$ & $\begin{array}{l}=e_{1} \\
=2 a e_{1} \\
=a e_{2}-e_{3} \\
=e_{2}+a e_{3}\end{array}$ & Разрешимая & $a>0$, нет & $y_{1}, y_{2}$ \\
\hline $\begin{array}{l}A_{4,12} \\
\text { Класс II }\end{array}$ & $\begin{array}{l}{\left[e_{1}, e_{3}\right]} \\
{\left[e_{2}, e_{3}\right]} \\
{\left[e_{1}, e_{4}\right]} \\
{\left[e_{2}, e_{4}\right]}\end{array}$ & $\begin{array}{l}=e_{1} \\
=e_{2} \\
=-e_{2} \\
=e_{1}\end{array}$ & Разрешимая & нет & $y_{1}, y_{2}$ \\
\hline \multicolumn{6}{|c|}{ Разложимые } \\
\hline $\begin{array}{l}A_{4,13} \\
\text { Класс II } \\
\end{array}$ & - & & Абелева & $x_{1}, x_{2}, x_{3}, x_{4}$ & $y_{1}, y_{2}, y_{3}, y_{4}$ \\
\hline $\begin{array}{l}A_{4,14} \\
\text { Класс II }\end{array}$ & {$\left[e_{1}, e_{2}\right]$} & $=e_{1}$ & Разрешимая & $x_{3}, x_{4}$ & $y_{2}, y_{3}, y_{4}$ \\
\hline $\begin{array}{l}A_{4,15} \\
\text { Класс II }\end{array}$ & $\begin{array}{l}{\left[e_{1}, e_{2}\right]} \\
{\left[e_{3}, e_{4}\right]}\end{array}$ & $\begin{array}{l}=e_{1} \\
=e_{3}\end{array}$ & Разрешимая & нет & $y_{2}, y_{3}$ \\
\hline $\begin{array}{l}A_{4,16} \\
\text { Класс II }\end{array}$ & {$\left[e_{2}, e_{3}\right]$} & $=e_{1}$ & Нильпотентная & $x_{1}, x_{4}$ & $y_{1}, y_{2}, y_{4}$ \\
\hline $\begin{array}{l}A_{4,17} \\
\text { Класс II }\end{array}$ & $\begin{array}{l}{\left[e_{1}, e_{3}\right]} \\
{\left[e_{2}, e_{3}\right]}\end{array}$ & $\begin{array}{l}=e_{1} \\
=e_{1}+e_{2}\end{array}$ & Разрешимая & $x_{1} \exp \left[-\frac{x_{2}}{x_{1}}\right], x_{4}$ & $y_{1}, y_{2}, y_{4}$ \\
\hline $\begin{array}{l}A_{4,18} \\
\text { Класс II }\end{array}$ & $\begin{array}{l}{\left[e_{1}, e_{3}\right]} \\
{\left[e_{2}, e_{3}\right]}\end{array}$ & $\begin{array}{l}=e_{1} \\
=e_{2}\end{array}$ & Разрешимая & $\frac{x_{2}}{x_{1}}, x_{4}$ & $y_{1}, y_{2}, y_{4}$ \\
\hline $\begin{array}{l}A_{4,19} \\
\text { Класс II }\end{array}$ & $\begin{array}{l}{\left[e_{1}, e_{3}\right]} \\
{\left[e_{2}, e_{3}\right]}\end{array}$ & $\begin{array}{l}=e_{1} \\
=-e_{2}\end{array}$ & Разрешимая & $x_{1} x_{2}, x_{4}$ & $y_{1}, y_{2}, y_{4}$ \\
\hline $\begin{array}{l}A_{4,20} \\
\text { Класс II }\end{array}$ & $\begin{array}{l}{\left[e_{1}, e_{3}\right]} \\
{\left[e_{2}, e_{3}\right]}\end{array}$ & $\begin{array}{l}=e_{1} \\
=a e_{2}\end{array}$ & Разрешимая & $0<|a|<1, x_{2} x_{1}^{-a}, x_{4}$ & $y_{1}, y_{2}, y_{4}$ \\
\hline $\begin{array}{l}A_{4,21} \\
\text { Класс II }\end{array}$ & $\begin{array}{l}{\left[e_{1}, e_{3}\right]} \\
{\left[e_{2}, e_{3}\right]}\end{array}$ & $\begin{array}{l}=-e_{2} \\
=e_{1}\end{array}$ & Разрешимая & $x_{1}^{2}+x_{2}^{2}, x_{4}$ & $y_{1}, y_{2}, y_{4}$ \\
\hline
\end{tabular}


Таблица 2 (продолжение)

\begin{tabular}{|l|l|l|l|l|}
\hline $\begin{array}{l}A_{4,22} \\
\text { Класс II }\end{array}$ & $\begin{array}{l}{\left[e_{1}, e_{3}\right]=a e_{1}-e_{2},} \\
{\left[e_{2}, e_{3}\right]=e_{1}+a e_{2}}\end{array}$ & Разрешимая & $\begin{array}{l}a>0, \\
\left(x_{1}^{2}+x_{2}^{2}\right)\left[\frac{x_{1}+i x_{2}}{x_{1}-i x_{2}}\right]^{i a}, x_{4}\end{array}$ & $y_{1}, y_{2}, y_{4}$ \\
\hline $\begin{array}{l}A_{4,23} \\
\text { Класс I }\end{array}$ & $\begin{array}{l}{\left[e_{1}, e_{3}\right]=-2 e_{2},} \\
{\left[e_{1}, e_{2}\right]=e_{1},} \\
{\left[e_{2}, e_{3}\right]=e_{3}}\end{array}$ & Редуктивная & $x_{2}^{2}+x_{1} x_{3}, x_{4}$ & $\begin{array}{l}\text { метод } \\
\text { сдвига } \\
\text { аргумента }\end{array}$ \\
\hline $\begin{array}{l}A_{4,24} \\
\text { Класс I }\end{array}$ & $\begin{array}{l}{\left[e_{1}, e_{2}\right]=e_{3},} \\
{\left[e_{2}, e_{3}\right]=e_{1},} \\
{\left[e_{3}, e_{1}\right]=e_{2}}\end{array}$ & Редуктивная & $x_{1}^{2}+x_{2}^{2}+x_{3}^{2}, x_{4}$ & $\begin{array}{l}\text { метод } \\
\text { сдвига } \\
\text { аргумента }\end{array}$ \\
\hline
\end{tabular}

ТАБлицА 3. Все пятимерные вещественные алгебры Ли

\begin{tabular}{|c|c|c|c|c|}
\hline \multicolumn{5}{|c|}{ Неразложсимые } \\
\hline $\begin{array}{l}A_{5,1} \\
\text { Класс II }\end{array}$ & $\begin{array}{l}{\left[e_{3}, e_{5}\right]=e_{1}} \\
{\left[e_{4}, e_{5}\right]=e_{2}}\end{array}$ & Нильпотентная & $x_{1}, x_{2}, x_{2} x_{3}-x_{1} x_{4}$ & $y_{1}, y_{2}, y_{3}, y_{4}$ \\
\hline $\begin{array}{l}A_{5,2} \\
\text { Класс II }\end{array}$ & $\begin{array}{l}{\left[e_{2}, e_{5}\right]=e_{1}} \\
{\left[e_{3}, e_{5}\right]=e_{2}} \\
{\left[e_{4}, e_{5}\right]=e_{3}}\end{array}$ & Нильпотентная & $\begin{array}{l}x_{1}, x_{2}^{2}-2 x_{1} x_{3}, \\
x_{2}^{3}+3 x_{1}^{2} x_{4}-3 x_{1} x_{2} x_{3}\end{array}$ & $y_{1}, y_{2}, y_{3}, y_{4}$ \\
\hline $\begin{array}{l}A_{5,3} \\
\text { Класс III }\end{array}$ & $\begin{array}{l}{\left[e_{3}, e_{4}\right]=e_{2}} \\
{\left[e_{3}, e_{5}\right]=e_{1}} \\
{\left[e_{4}, e_{5}\right]=e_{3}}\end{array}$ & Нильпотентная & $x_{1}, x_{2}, x_{3}^{2}+2 x_{2} x_{5}-2 x_{1} x_{4}$ & $\begin{array}{l}y_{1}, y_{2}, y_{3} \\
y_{1} y_{4}-y_{2} y_{5}\end{array}$ \\
\hline $\begin{array}{l}A_{5,4} \\
\text { Класс II }\end{array}$ & $\begin{array}{l}{\left[e_{2}, e_{4}\right]=e_{1}} \\
{\left[e_{3}, e_{5}\right]=e_{1}}\end{array}$ & Нильпотентная & $x_{1}$ & $y_{1}, y_{2}, y_{3}$ \\
\hline $\begin{array}{l}A_{5,5} \\
\text { Класс II }\end{array}$ & $\begin{array}{l}{\left[e_{3}, e_{4}\right]=e_{1}} \\
{\left[e_{2}, e_{5}\right]=e_{1}} \\
{\left[e_{3}, e_{5}\right]=e_{2}}\end{array}$ & Нильпотентная & $x_{1}$ & $y_{1}, y_{2}, y_{3}$ \\
\hline $\begin{array}{l}A_{5,6} \\
\text { Класс II }\end{array}$ & $\begin{array}{l}{\left[e_{3}, e_{4}\right]=e_{1}} \\
{\left[e_{2}, e_{5}\right]=e_{1}} \\
{\left[e_{3}, e_{5}\right]=e_{2}} \\
{\left[e_{4}, e_{5}\right]=e_{3}}\end{array}$ & Нильпотентная & $x_{1}$ & $y_{1}, y_{2}, y_{3}$ \\
\hline $\begin{array}{l}A_{5,7} \\
\text { Класс II }\end{array}$ & $\begin{array}{l}{\left[e_{1}, e_{5}\right]=e_{1}} \\
{\left[e_{2}, e_{5}\right]=a e_{2}} \\
{\left[e_{3}, e_{5}\right]=b e_{3}} \\
{\left[e_{4}, e_{5}\right]=c e_{4}}\end{array}$ & Разрешимая & $\begin{array}{l}a b c \neq 0, \\
-1 \leqslant c \leqslant b \leqslant a \leqslant 1 \\
\frac{x_{1}^{a}}{x_{2}}, \frac{x_{1}^{b}}{x_{3}}, \frac{x_{1}^{c}}{x_{4}}\end{array}$ & $y_{1}, y_{2}, y_{3}, y_{4}$ \\
\hline $\begin{array}{l}A_{5,8} \\
\text { Класс II }\end{array}$ & $\begin{array}{l}{\left[e_{2}, e_{5}\right]=e_{1}} \\
{\left[e_{3}, e_{5}\right]=e_{3}} \\
{\left[e_{4}, e_{5}\right]=c e_{4}}\end{array}$ & Разрешимая & $\begin{array}{l}0<|c| \leqslant 1, \\
x_{1}, \frac{x_{3}^{c}}{x_{4}}, x_{3} \exp \left(-\frac{x_{2}}{x_{1}}\right)\end{array}$ & $y_{1}, y_{2}, y_{3}, y_{4}$ \\
\hline $\begin{array}{l}A_{5,9} \\
\text { Класс II }\end{array}$ & $\begin{array}{l}{\left[e_{1}, e_{5}\right]=e_{1},} \\
{\left[e_{2}, e_{5}\right]=e_{1}+e_{2},} \\
{\left[e_{3}, e_{5}\right]=b e_{3},} \\
{\left[e_{4}, e_{5}\right]=c e_{4}}\end{array}$ & Разрешимая & $\begin{array}{l}0 \neq c \leqslant b, \frac{x_{1}^{b}}{x_{3}}, \frac{x_{1}^{c}}{x_{4}} \\
x_{1} \exp \left(-\frac{x_{2}}{x_{1}}\right)\end{array}$ & $y_{1}, y_{2}, y_{3}, y_{4}$ \\
\hline $\begin{array}{l}A_{5,10} \\
\text { Класс II }\end{array}$ & $\begin{array}{l}{\left[e_{2}, e_{5}\right]=e_{1}} \\
{\left[e_{3}, e_{5}\right]=e_{2}} \\
{\left[e_{4}, e_{5}\right]=e_{4}}\end{array}$ & Разрешимая & $\begin{array}{l}x_{1}, x_{2}^{2}-2 x_{1} x_{3} \\
x_{4} \exp \left(-\frac{x_{2}}{x_{1}}\right)\end{array}$ & $y_{1}, y_{2}, y_{3}, y_{4}$ \\
\hline $\begin{array}{l}A_{5,11} \\
\text { Класс II }\end{array}$ & $\begin{array}{l}{\left[e_{1}, e_{5}\right]=e_{1},} \\
{\left[e_{2}, e_{5}\right]=e_{1}+e_{2},} \\
{\left[e_{3}, e_{5}\right]=e_{2}+e_{3},} \\
{\left[e_{4}, e_{5}\right]=c e_{4}}\end{array}$ & Разрешимая & $\begin{array}{l}c \neq 0, \frac{x_{1}^{c}}{x_{4}}, x_{1} \exp \left(-\frac{x_{2}}{x_{1}}\right), \\
2 \frac{x_{3}}{x_{1}}-\frac{x_{2}^{2}}{x_{1}^{2}}\end{array}$ & $y_{1}, y_{2}, y_{3}, y_{4}$ \\
\hline
\end{tabular}




\section{Таблица 3 (продолжение)}

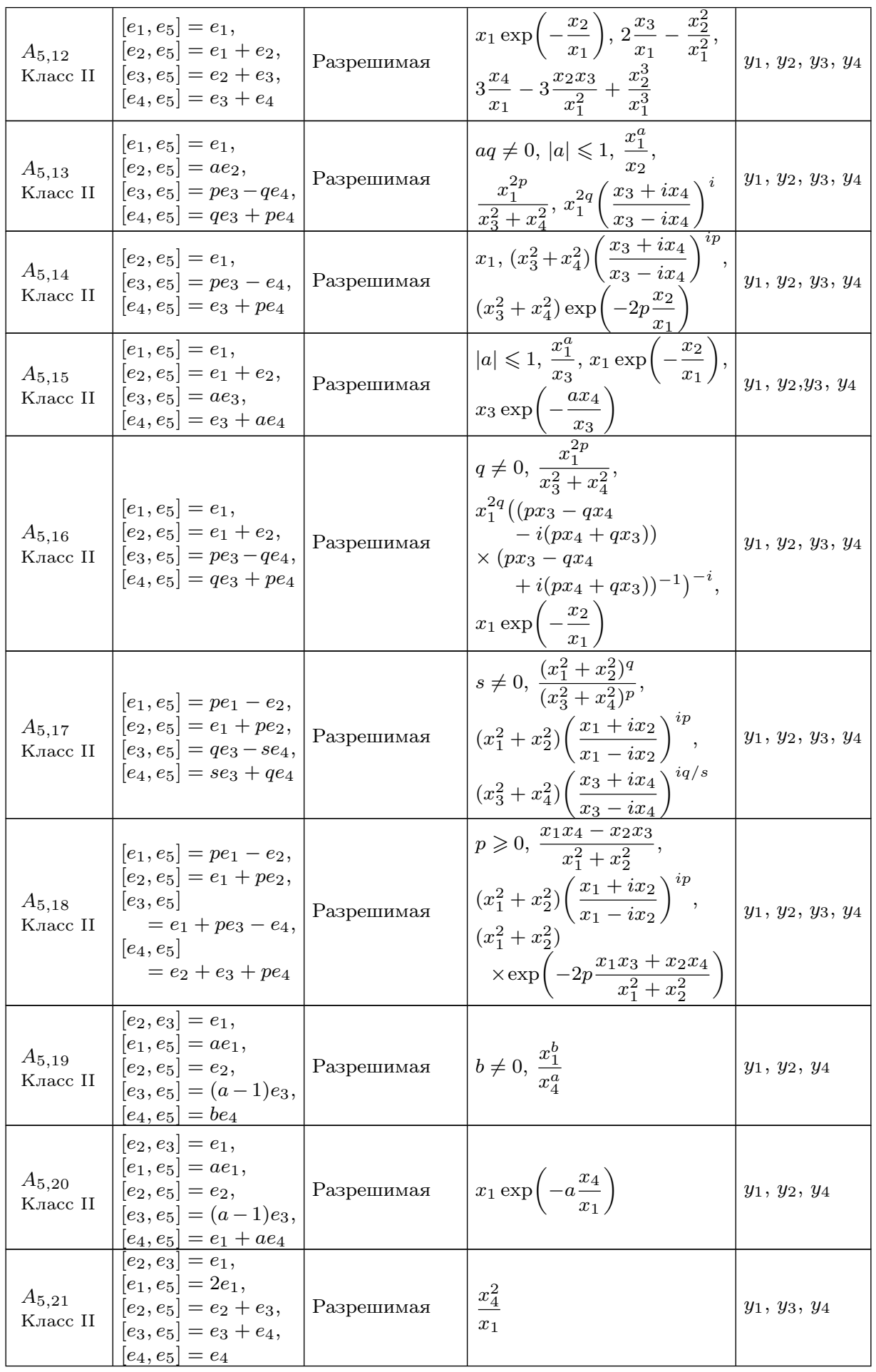


Таблица 3 (продолжение)

\begin{tabular}{|c|c|c|c|c|c|}
\hline $\begin{array}{l}A_{5,22} \\
\text { Класс II }\end{array}$ & $\begin{array}{l}{\left[e_{2}, e_{3}\right]} \\
{\left[e_{2}, e_{5}\right]} \\
{\left[e_{4}, e_{5}\right]}\end{array}$ & $\begin{array}{l}=e_{1} \\
=e_{3} \\
=e_{4}\end{array}$ & Разрешимая & $x_{1}$ & $y_{1}, y_{3}, y_{4}$ \\
\hline $\begin{array}{l}A_{5,23} \\
\text { Класс II }\end{array}$ & $\begin{array}{l}{\left[e_{2}, e_{3}\right]} \\
{\left[e_{1}, e_{5}\right]} \\
{\left[e_{2}, e_{5}\right]} \\
{\left[e_{3}, e_{5}\right]} \\
{\left[e_{4}, e_{5}\right]}\end{array}$ & $\begin{array}{l}=e_{1}, \\
=2 e_{1}, \\
=e_{2}+e_{3}, \\
=e_{3}, \\
=b e_{4}\end{array}$ & Разрешимая & $b \neq 0, \frac{x_{1}^{b}}{x_{4}^{2}}$ & $y_{1}, y_{3}, y_{4}$ \\
\hline $\begin{array}{l}A_{5,24} \\
\text { Класс II }\end{array}$ & $\begin{array}{l}{\left[e_{2}, e_{3}\right]} \\
{\left[e_{1}, e_{5}\right]} \\
{\left[e_{2}, e_{5}\right]} \\
{\left[e_{3}, e_{5}\right]} \\
{\left[e_{4}, e_{5}\right]} \\
\end{array}$ & $\begin{array}{l}=e_{1}, \\
=2 e_{1}, \\
=e_{2}+e_{3}, \\
=e_{3}, \\
=\varepsilon e_{1}+2 e_{4}\end{array}$ & Разрешимая & $\varepsilon= \pm 1, x_{1} \exp \left[-2 \varepsilon\left(\frac{x_{4}}{x_{1}}\right)\right]$ & $y_{1}, y_{3}, y_{4}$ \\
\hline $\begin{array}{l}A_{5,25} \\
\text { Класс IV }\end{array}$ & $\begin{array}{l}{\left[e_{2}, e_{3}\right]} \\
{\left[e_{1}, e_{5}\right]} \\
{\left[e_{2}, e_{5}\right]} \\
{\left[e_{3}, e_{5}\right]} \\
{\left[e_{4}, e_{5}\right]}\end{array}$ & $\begin{array}{l}=e_{1}, \\
=2 p e_{1}, \\
=p e_{2}+e_{3}, \\
=p e_{3}-e_{2}, \\
=b e_{4}\end{array}$ & Разрешимая & $b \neq 0, \frac{x_{1}^{b}}{x_{4}^{2 p}}$ & $y_{1}, y_{2}, y_{4}$ \\
\hline $\begin{array}{l}A_{5,26} \\
\text { Класс IV }\end{array}$ & $\begin{array}{r}{\left[e_{2}, e_{3}\right]} \\
{\left[e_{1}, e_{5}\right]} \\
{\left[e_{2}, e_{5}\right]} \\
{\left[e_{3}, e_{5}\right]} \\
{\left[e_{4}, e_{5}\right]} \\
\quad=\varepsilon \epsilon\end{array}$ & $\begin{array}{l}=e_{1}, \\
=2 p e_{1}, \\
=p e_{2}+e_{3}, \\
=p e_{3}-e_{2}, \\
e_{1}+2 p e_{4}\end{array}$ & Разрешимая & $\varepsilon= \pm 1, x_{1} \exp \left[-2 \varepsilon p \frac{x_{4}}{x_{1}}\right]$ & $y_{1}, y_{2}, y_{4}$ \\
\hline $\begin{array}{l}A_{5,27} \\
\text { Класс II }\end{array}$ & $\begin{array}{l}{\left[e_{2}, e_{3}\right]} \\
{\left[e_{1}, e_{5}\right]} \\
{\left[e_{3}, e_{5}\right]} \\
{\left[e_{4}, e_{5}\right]}\end{array}$ & $\begin{array}{l}=e_{1} \\
=e_{1} \\
=e_{3}+e_{4} \\
=e_{1}+e_{4}\end{array}$ & Разрешимая & $x_{1} \exp \left(-\frac{x_{4}}{x_{1}}\right)$ & $y_{1}, y_{3}, y_{4}$ \\
\hline $\begin{array}{l}A_{5,28} \\
\text { Класс II }\end{array}$ & $\begin{array}{l}{\left[e_{2}, e_{3}\right]} \\
{\left[e_{1}, e_{5}\right]} \\
{\left[e_{2}, e_{5}\right]} \\
{\left[e_{3}, e_{5}\right]} \\
{\left[e_{4}, e_{5}\right]}\end{array}$ & $\begin{array}{l}=e_{1}, \\
=a e_{1}, \\
=(a-1) e_{2}, \\
=e_{3}+e_{4}, \\
=e_{4}\end{array}$ & Разрешимая & $\frac{x_{4}^{a}}{x_{1}}$ & $y_{1}, y_{3}, y_{4}$ \\
\hline $\begin{array}{l}A_{5,29} \\
\text { Класс II }\end{array}$ & $\begin{array}{l}{\left[e_{2}, e_{3}\right]} \\
{\left[e_{1}, e_{5}\right]} \\
{\left[e_{2}, e_{5}\right]} \\
{\left[e_{3}, e_{5}\right]}\end{array}$ & $\begin{array}{l}=e_{1} \\
=e_{1} \\
=e_{2} \\
=e_{4}\end{array}$ & Разрешимая & $x_{4}$ & $y_{1}, y_{2}, y_{4}$ \\
\hline $\begin{array}{l}A_{5,30} \\
\text { Класс II }\end{array}$ & $\begin{array}{l}{\left[e_{2}, e_{4}\right]} \\
{\left[e_{3}, e_{4}\right]} \\
{\left[e_{1}, e_{5}\right]} \\
{\left[e_{2}, e_{5}\right]} \\
{\left[e_{3}, e_{5}\right]} \\
{\left[e_{4}, e_{5}\right]}\end{array}$ & $\begin{array}{l}=e_{1}, \\
=e_{2}, \\
=(a+1) e_{1}, \\
=a e_{2}, \\
=(a-1) e_{3}, \\
=e_{4}\end{array}$ & Разрешимая & $\frac{\left(x_{2}^{2}-2 x_{1} x_{3}\right)^{a+1}}{x_{1}^{2 a}}$ & $y_{1}, y_{2}, y_{3}$ \\
\hline $\begin{array}{l}A_{5,31} \\
\text { Класс II }\end{array}$ & $\begin{array}{l}{\left[e_{2}, e_{4}\right]} \\
{\left[e_{3}, e_{4}\right]} \\
{\left[e_{1}, e_{5}\right]} \\
{\left[e_{2}, e_{5}\right]} \\
{\left[e_{3}, e_{5}\right]} \\
{\left[e_{4}, e_{5}\right]}\end{array}$ & $\begin{array}{l}=e_{1}, \\
=e_{2}, \\
=3 e_{1}, \\
=2 e_{2}, \\
=e_{3}, \\
=e_{3}+e_{4}\end{array}$ & Разрешимая & $\frac{\left(x_{2}^{2}-2 x_{1} x_{3}\right)^{3}}{x_{1}^{4}}$ & $y_{1}, y_{2}, y_{3}$ \\
\hline $\begin{array}{l}A_{5,32} \\
\text { Класс II }\end{array}$ & $\begin{array}{l}{\left[e_{2}, e_{4}\right]} \\
{\left[e_{3}, e_{4}\right]} \\
{\left[e_{1}, e_{5}\right]} \\
{\left[e_{2}, e_{5}\right]} \\
{\left[e_{3}, e_{5}\right]}\end{array}$ & $\begin{array}{l}=e_{1}, \\
=e_{2}, \\
=e_{1}, \\
=e_{2}, \\
=a e_{1}+e_{3}\end{array}$ & Разрешимая & $x_{1}^{2 a} \exp \left[\frac{x_{2}^{2}-2 x_{1} x_{3}}{x_{1}^{2}}\right]$ & $y_{1}, y_{2}, y_{3}$ \\
\hline
\end{tabular}


Таблица 3 (продолжение)

\begin{tabular}{|c|c|c|c|c|c|}
\hline $\begin{array}{l}A_{5,33} \\
\text { Класс II }\end{array}$ & $\begin{array}{l}{\left[e_{1}, e_{4}\right]} \\
{\left[e_{3}, e_{4}\right]} \\
{\left[e_{2}, e_{5}\right]} \\
{\left[e_{3}, e_{5}\right]}\end{array}$ & $\begin{array}{l}=e_{1} \\
=b e_{3} \\
=e_{2} \\
=a e_{3}\end{array}$ & Разрешимая & $a^{2}+b^{2} \neq 0, \frac{x_{1}^{b} x_{2}^{a}}{x_{3}}$ & $y_{1}, y_{2}, y_{3}$ \\
\hline $\begin{array}{l}A_{5,34} \\
\text { Класс II }\end{array}$ & $\begin{array}{l}{\left[e_{1}, e_{4}\right]} \\
{\left[e_{2}, e_{4}\right]} \\
{\left[e_{3}, e_{4}\right]} \\
{\left[e_{1}, e_{5}\right]} \\
{\left[e_{3}, e_{5}\right]}\end{array}$ & $\begin{array}{l}=a e_{1} \\
=e_{2} \\
=e_{3} \\
=e_{1} \\
=e_{2}\end{array}$ & Разрешимая & $\frac{x_{2}^{a}}{x_{1}} \exp \left(\frac{x_{3}}{x_{2}}\right)$ & $y_{1}, y_{2}, y_{3}$ \\
\hline $\begin{array}{l}A_{5,35} \\
\text { Класс II }\end{array}$ & $\begin{array}{l}{\left[e_{1}, e_{4}\right]} \\
{\left[e_{2}, e_{4}\right]} \\
{\left[e_{3}, e_{4}\right]} \\
{\left[e_{1}, e_{5}\right]} \\
{\left[e_{2}, e_{5}\right]} \\
{\left[e_{3}, e_{5}\right]}\end{array}$ & $\begin{array}{l}=b e_{1}, \\
=e_{2}, \\
=e_{3} \\
=a e_{1} \\
=-e_{3} \\
=e_{2}\end{array}$ & Разрешимая & $\begin{array}{l}a^{2}+b^{2} \neq 0, \\
\frac{x_{1}^{2}}{\left(x_{2}^{2}+x_{3}^{2}\right)^{b}}\left(\frac{x_{2}+i x_{3}}{x_{2}-i x_{3}}\right)^{i a}\end{array}$ & $y_{1}, y_{2}, y_{3}$ \\
\hline $\begin{array}{l}A_{5,36} \\
\text { Класс ІІІ }\end{array}$ & $\begin{array}{l}{\left[e_{2}, e_{3}\right]} \\
{\left[e_{1}, e_{4}\right]} \\
{\left[e_{2}, e_{4}\right]} \\
{\left[e_{2}, e_{5}\right]} \\
{\left[e_{3}, e_{5}\right]}\end{array}$ & $\begin{array}{l}=e_{1}, \\
=e_{1}, \\
=e_{2}, \\
=-e_{2}, \\
=e_{3}\end{array}$ & Разрешимая & $\frac{x_{2} x_{3}+x_{1} x_{5}}{x_{1}}$ & $\begin{array}{l}y_{1}, y_{2} \\
y_{2} y_{3}+y_{1} y_{5}\end{array}$ \\
\hline $\begin{array}{l}A_{5,37} \\
\text { Класс VI }\end{array}$ & $\begin{array}{l}{\left[e_{2}, e_{3}\right]} \\
{\left[e_{1}, e_{4}\right]} \\
{\left[e_{2}, e_{4}\right]} \\
{\left[e_{3}, e_{4}\right]} \\
{\left[e_{2}, e_{5}\right]} \\
{\left[e_{3}, e_{5}\right]}\end{array}$ & $\begin{array}{l}=e_{1}, \\
=2 e_{1}, \\
=e_{2}, \\
=e_{3}, \\
=-e_{3}, \\
=e_{2}\end{array}$ & Разрешимая & $\frac{x_{2}^{2}+x_{3}^{2}+2 x_{1} x_{5}}{x_{1}}$ & $\begin{array}{l}y_{1}, y_{2}, \\
y_{1} y_{5} \\
+\frac{1}{2}\left(y_{2}^{2}+y_{3}^{2}\right)\end{array}$ \\
\hline $\begin{array}{l}A_{5,38} \\
\text { Класс II }\end{array}$ & $\begin{array}{l}{\left[e_{1}, e_{4}\right]} \\
{\left[e_{2}, e_{5}\right]} \\
{\left[e_{4}, e_{5}\right]}\end{array}$ & $\begin{array}{l}=e_{1} \\
=e_{2} \\
=e_{3}\end{array}$ & Разрешимая & $x_{3}$ & $y_{1}, y_{2}, y_{3}$ \\
\hline $\begin{array}{l}A_{5,39} \\
\text { Класс II }\end{array}$ & $\begin{array}{l}{\left[e_{1}, e_{4}\right]} \\
{\left[e_{2}, e_{4}\right]} \\
{\left[e_{1}, e_{5}\right]} \\
{\left[e_{2}, e_{5}\right]} \\
{\left[e_{4}, e_{5}\right]}\end{array}$ & $\begin{array}{l}=e_{1}, \\
=e_{2}, \\
=-e_{2}, \\
=e_{1}, \\
=e_{3}\end{array}$ & Разрешимая & $x_{3}$ & $y_{1}, y_{2}, y_{3}$ \\
\hline $\begin{array}{l}A_{5,40} \\
\text { Класс III }\end{array}$ & \begin{tabular}{|l}
{$\left[e_{1}, e_{2}\right]$} \\
{$\left[e_{1}, e_{3}\right]$} \\
{$\left[e_{2}, e_{3}\right]$} \\
{$\left[e_{1}, e_{4}\right]$} \\
{$\left[e_{2}, e_{4}\right]$} \\
{$\left[e_{2}, e_{5}\right]$} \\
{$\left[e_{3}, e_{5}\right]$}
\end{tabular} & $\begin{array}{l}=2 e_{1}, \\
=-e_{2}, \\
=2 e_{3}, \\
=e_{5}, \\
=e_{4}, \\
=-e_{5}, \\
=e_{4}\end{array}$ & Общего типа & $x_{1} x_{4}^{2}-x_{2} x_{4} x_{5}-x_{3} x_{5}^{2}$ & $\begin{array}{l}y_{2} y_{4} y_{5}-y_{1} y_{4}^{2} \\
+y_{3} y_{5}^{2}, \quad y_{4}, \\
y_{5}\end{array}$ \\
\hline \multicolumn{6}{|c|}{ Разложимые } \\
\hline $\begin{array}{l}A_{5,41} \\
\text { Класс II }\end{array}$ & - & & Абелева & $x_{1}, x_{2}, x_{3}, x_{4}, x_{5}$ & $\begin{array}{l}y_{1}, y_{2}, \quad y_{3} \\
y_{4}, y_{5}\end{array}$ \\
\hline $\begin{array}{l}A_{5,42} \\
\text { Класс II }\end{array}$ & {$\left[e_{1}, e_{2}\right]$} & $=e_{1}$ & Разрешимая & $x_{3}, x_{4}, x_{5}$ & $y_{2}, y_{3}, y_{4}, y_{5}$ \\
\hline $\begin{array}{l}A_{5,43} \\
\text { Класс II }\end{array}$ & $\begin{array}{l}{\left[e_{1}, e_{2}\right]} \\
{\left[e_{3}, e_{4}\right]} \\
\end{array}$ & $\begin{array}{l}=e_{1} \\
=e_{3}\end{array}$ & Разрешимая & $x_{5}$ & $y_{2}, y_{3}, y_{5}$ \\
\hline $\begin{array}{l}A_{5,44} \\
\text { Класс II }\end{array}$ & {$\left[e_{2}, e_{3}\right]$} & $=e_{1}$ & Нильпотентная & $x_{1}, x_{4}, x_{5}$ & $y_{1}, y_{2}, y_{3}, y_{4}$ \\
\hline $\begin{array}{l}A_{5,45} \\
\text { Класс II }\end{array}$ & $\begin{array}{l}{\left[e_{1}, e_{3}\right]} \\
{\left[e_{2}, e_{3}\right]}\end{array}$ & $\begin{array}{l}=e_{1} \\
=e_{1}+e_{2}\end{array}$ & Разрешимая & $x_{1} \exp \left[-\frac{x_{2}}{x_{1}}\right], x_{4}, x_{5}$ & $y_{1}, y_{2}, y_{4}, y_{5}$ \\
\hline
\end{tabular}


Таблица 3 (продолжение)

\begin{tabular}{|c|c|c|c|c|}
\hline $\begin{array}{l}A_{5,46} \\
\text { Класс II }\end{array}$ & $\begin{array}{l}{\left[e_{1}, e_{3}\right]=e_{1}} \\
{\left[e_{2}, e_{3}\right]=e_{2}}\end{array}$ & Разрешимая & $\frac{x_{2}}{x_{1}}, x_{4}, x_{5}$ & $y_{1}, y_{2}, y_{4}, y_{5}$ \\
\hline $\begin{array}{l}A_{5,47} \\
\text { Класс II }\end{array}$ & $\begin{array}{l}{\left[e_{1}, e_{3}\right]=e_{1}} \\
{\left[e_{2}, e_{3}\right]=-e_{2}}\end{array}$ & Разрешимая & $x_{1} x_{2}, x_{4}, x_{5}$ & $y_{1}, y_{2}, y_{4}, y_{5}$ \\
\hline $\begin{array}{l}A_{5,48} \\
\text { Класс II }\end{array}$ & $\begin{array}{l}{\left[e_{1}, e_{3}\right]=e_{1}} \\
{\left[e_{2}, e_{3}\right]=a e_{2}}\end{array}$ & Разрешимая & $0<|a|<1, x_{2} x_{1}^{-a}, x_{4}, x_{5}$ & $y_{1}, y_{2}, y_{4}, y_{5}$ \\
\hline $\begin{array}{l}A_{5,49} \\
\text { Класс II }\end{array}$ & $\begin{array}{l}{\left[e_{1}, e_{3}\right]=-e_{2}} \\
{\left[e_{2}, e_{3}\right]=e_{1}}\end{array}$ & Разрешимая & $x_{1}^{2}+x_{2}^{2}, x_{4}, x_{5}$ & $y_{1}, y_{2}, y_{4}, y_{5}$ \\
\hline $\begin{array}{l}A_{5,50} \\
\text { Класс II }\end{array}$ & $\begin{array}{l}{\left[e_{1}, e_{3}\right]=a e_{1}-e_{2}} \\
{\left[e_{2}, e_{3}\right]=e_{1}+a e_{2}}\end{array}$ & Разрешимая & $\begin{array}{l}a>0 \\
\left(x_{1}^{2}+x_{2}^{2}\right)\left[\frac{x_{1}+i x_{2}}{x_{1}-i x_{2}}\right]^{i a} \\
x_{4}, x_{5}\end{array}$ & $y_{1}, y_{2}, y_{4}, y_{5}$ \\
\hline $\begin{array}{l}A_{5,51} \\
\text { Класс I }\end{array}$ & $\begin{array}{l}{\left[e_{1}, e_{3}\right]=-2 e_{2}} \\
{\left[e_{1}, e_{2}\right]=e_{1}} \\
{\left[e_{2}, e_{3}\right]=e_{3}}\end{array}$ & Редуктивная & $x_{2}^{2}+x_{1} x_{3}, x_{4}, x_{5}$ & $\begin{array}{l}\text { метод } \\
\text { сдвига } \\
\text { аргумента }\end{array}$ \\
\hline $\begin{array}{l}A_{5,52} \\
\text { Класс I }\end{array}$ & $\begin{array}{l}{\left[e_{1}, e_{2}\right]=e_{3}} \\
{\left[e_{2}, e_{3}\right]=e_{1}} \\
{\left[e_{3}, e_{1}\right]=e_{2}}\end{array}$ & Редуктивная & $x_{1}^{2}+x_{2}^{2}+x_{3}^{2}, x_{4}, x_{5}$ & $\begin{array}{l}\text { метод } \\
\text { сдвига } \\
\text { аргумента }\end{array}$ \\
\hline $\begin{array}{l}A_{5,53} \\
\text { Класс II }\end{array}$ & $\begin{array}{l}{\left[e_{2}, e_{3}\right]=e_{1}} \\
{\left[e_{4}, e_{5}\right]=e_{4}}\end{array}$ & Разрешимая & $x_{1}$ & $y_{1}, y_{2}, y_{4}$ \\
\hline $\begin{array}{l}A_{5,54} \\
\text { Класс II }\end{array}$ & $\begin{array}{l}{\left[e_{1}, e_{3}\right]=e_{1}} \\
{\left[e_{2}, e_{3}\right]=e_{1}+e_{2},} \\
{\left[e_{4}, e_{5}\right]=e_{4}}\end{array}$ & Разрешимая & $x_{1} \exp \left[-\frac{x_{2}}{x_{1}}\right]$ & $y_{1}, y_{2}, y_{4}$ \\
\hline $\begin{array}{l}A_{5,55} \\
\text { Класс II }\end{array}$ & $\begin{array}{l}{\left[e_{1}, e_{3}\right]=e_{1}} \\
{\left[e_{2}, e_{3}\right]=e_{2}} \\
{\left[e_{4}, e_{5}\right]=e_{4}}\end{array}$ & Разрешимая & $\frac{x_{2}}{x_{1}}$ & $y_{1}, y_{2}, y_{4}$ \\
\hline $\begin{array}{l}A_{5,56} \\
\text { Класс II }\end{array}$ & $\begin{array}{l}{\left[e_{1}, e_{3}\right]=e_{1}} \\
{\left[e_{2}, e_{3}\right]=-e_{2}} \\
{\left[e_{4}, e_{5}\right]=e_{4}}\end{array}$ & Разрешимая & $x_{1} x_{2}$ & $y_{1}, y_{2}, y_{4}$ \\
\hline $\begin{array}{l}A_{5,57} \\
\text { Класс II }\end{array}$ & $\begin{array}{l}{\left[e_{1}, e_{3}\right]=e_{1}} \\
{\left[e_{2}, e_{3}\right]=a e_{2}} \\
{\left[e_{4}, e_{5}\right]=e_{4}}\end{array}$ & Разрешимая & $0<|a|<1, x_{2} x_{1}^{-a}$ & $y_{1}, y_{2}, y_{4}$ \\
\hline $\begin{array}{l}A_{5,58} \\
\text { Класс II }\end{array}$ & $\begin{array}{l}{\left[e_{1}, e_{3}\right]=-e_{2}} \\
{\left[e_{2}, e_{3}\right]=e_{1}} \\
{\left[e_{4}, e_{5}\right]=e_{4}}\end{array}$ & Разрешимая & $x_{1}^{2}+x_{2}^{2}$ & $y_{1}, y_{2}, y_{4}$ \\
\hline $\begin{array}{l}A_{5,59} \\
\text { Класс II }\end{array}$ & $\begin{array}{l}{\left[e_{1}, e_{3}\right]=a e_{1}-e_{2},} \\
{\left[e_{2}, e_{3}\right]=e_{1}+a e_{2},} \\
{\left[e_{4}, e_{5}\right]=e_{4}}\end{array}$ & Разрешимая & $\begin{array}{l}a>0, \\
\left(x_{1}^{2}+x_{2}^{2}\right)\left[\frac{x_{1}+i x_{2}}{x_{1}-i x_{2}}\right]^{i a}\end{array}$ & $y_{1}, y_{2}, y_{4}$ \\
\hline $\begin{array}{l}A_{5,60} \\
\text { Класс } \\
\text { VII }\end{array}$ & $\begin{array}{l}{\left[e_{1}, e_{3}\right]=-2 e_{2}} \\
{\left[e_{1}, e_{2}\right]=e_{1}} \\
{\left[e_{2}, e_{3}\right]=e_{3}} \\
{\left[e_{4}, e_{5}\right]=e_{4}}\end{array}$ & Общего типа & $x_{2}^{2}+x_{1} x_{3}$ & $\begin{array}{l}y_{4}, \text { метод } \\
\text { сдвига } \\
\text { аргумента }\end{array}$ \\
\hline $\begin{array}{l}A_{5,61} \\
\text { Класс } \\
\text { VII }\end{array}$ & $\begin{array}{l}{\left[e_{1}, e_{2}\right]=e_{3}} \\
{\left[e_{2}, e_{3}\right]=e_{1}} \\
{\left[e_{3}, e_{1}\right]=e_{2}} \\
{\left[e_{4}, e_{5}\right]=e_{4}}\end{array}$ & Общего типа & $x_{1}^{2}+x_{2}^{2}+x_{3}^{2}$ & $\begin{array}{l}y_{4}, \text { метод } \\
\text { сдвига } \\
\text { аргумента }\end{array}$ \\
\hline $\begin{array}{l}A_{5,62} \\
\text { Класс II }\end{array}$ & $\begin{array}{l}{\left[e_{2}, e_{4}\right]=e_{1}} \\
{\left[e_{3}, e_{4}\right]=e_{2}}\end{array}$ & Нильпотентная & $x_{1}, x_{2}^{2}-2 x_{1} x_{3}, x_{5}$ & $y_{1}, y_{2}, y_{3}, y_{5}$ \\
\hline $\begin{array}{l}A_{5,63} \\
\text { Класс II }\end{array}$ & $\begin{array}{l}{\left[e_{1}, e_{4}\right]=a e_{1},} \\
{\left[e_{2}, e_{4}\right]=e_{2},} \\
{\left[e_{3}, e_{4}\right]=e_{2}+e_{3}}\end{array}$ & Разрешимая & $\begin{array}{l}a \neq 0, \\
x_{2} \exp \left(-\frac{x_{3}}{x_{2}}\right), \frac{x_{2}^{a}}{x_{1}}, x_{5}\end{array}$ & $y_{1}, y_{2}, y_{3}, y_{5}$ \\
\hline
\end{tabular}




\section{Таблица 3 (продолжение)}

\begin{tabular}{|c|c|c|c|c|}
\hline $\begin{array}{l}A_{5,64} \\
\text { Класс II }\end{array}$ & $\begin{array}{l}{\left[e_{1}, e_{4}\right]=e_{1}} \\
{\left[e_{3}, e_{4}\right]=e_{2}}\end{array}$ & Разрешимая & $x_{2}, x_{1} \exp \left(-\frac{x_{3}}{x_{2}}\right), x_{5}$ & $y_{1}, y_{2}, y_{3}, y_{5}$ \\
\hline $\begin{array}{l}A_{5,65} \\
\text { Класс II }\end{array}$ & $\begin{array}{l}{\left[e_{1}, e_{4}\right]=e_{1}} \\
{\left[e_{2}, e_{4}\right]=e_{1}+e_{2}} \\
{\left[e_{3}, e_{4}\right]=e_{2}+e_{3}}\end{array}$ & Разрешимая & $\begin{array}{l}x_{1} \exp \left(-\frac{x_{2}}{x_{1}}\right) \\
\frac{2 x_{1} x_{3}-x_{2}^{2}}{x_{1}^{2}}, x_{5}\end{array}$ & $y_{1}, y_{2}, y_{3}, y_{5}$ \\
\hline $\begin{array}{l}A_{5,66} \\
\text { Класс II }\end{array}$ & $\begin{array}{l}{\left[e_{1}, e_{4}\right]=e_{1}} \\
{\left[e_{2}, e_{4}\right]=a e_{2}} \\
{\left[e_{3}, e_{4}\right]=b e_{3}}\end{array}$ & Разрешимая & $\begin{array}{l}a b \neq 0,-1 \leqslant b \leqslant a \leqslant 1, \\
\frac{x_{1}^{a}}{x_{2}}, \frac{x_{1}^{b}}{x_{3}}, x_{5}\end{array}$ & $y_{1}, y_{2}, y_{3}, y_{5}$ \\
\hline $\begin{array}{l}A_{5,67} \\
\text { Класс II }\end{array}$ & $\begin{array}{l}{\left[e_{1}, e_{4}\right]=a e_{1}} \\
{\left[e_{2}, e_{4}\right]=b e_{2}-e_{3}} \\
{\left[e_{3}, e_{4}\right]=e_{2}+b e_{3}}\end{array}$ & Разрешимая & $\begin{array}{l}a \neq 0, b \geqslant 0, \frac{x_{1}^{2 b / a}}{x_{2}^{2}+x_{3}^{2}}, \\
\left(x_{2}^{2}+x_{3}^{2}\right)\left(\frac{x_{2}+i x_{3}}{x_{2}-i x_{3}}\right)^{i b}, x_{5}\end{array}$ & $y_{1}, y_{2}, y_{3}, y_{5}$ \\
\hline $\begin{array}{l}A_{5,68} \\
\text { Класс II }\end{array}$ & $\begin{array}{l}{\left[e_{2}, e_{3}\right]=e_{1}} \\
{\left[e_{1}, e_{4}\right]=2 e_{1}} \\
{\left[e_{2}, e_{4}\right]=e_{2}} \\
{\left[e_{3}, e_{4}\right]=e_{2}+e_{3}}\end{array}$ & Разрешимая & $x_{5}$ & $y_{1}, y_{2}, y_{5}$ \\
\hline $\begin{array}{l}A_{5,69} \\
\text { Класс III }\end{array}$ & $\begin{array}{l}{\left[e_{2}, e_{3}\right]=e_{1}} \\
{\left[e_{2}, e_{4}\right]=e_{2}} \\
{\left[e_{3}, e_{4}\right]=-e_{3}}\end{array}$ & Разрешимая & $x_{1}, x_{2} x_{3}-x_{1} x_{4}, x_{5}$ & $\begin{array}{l}y_{1}, y_{2}, \\
y_{2} y_{3}-y_{1} y_{4}, \\
y_{5}\end{array}$ \\
\hline $\begin{array}{l}A_{5,70} \\
\text { Класс II }\end{array}$ & $\begin{array}{l}{\left[e_{2}, e_{3}\right]=e_{1},} \\
{\left[e_{1}, e_{4}\right]=(1+b) e_{1},} \\
{\left[e_{2}, e_{4}\right]=e_{2},} \\
{\left[e_{3}, e_{4}\right]=b e_{3}}\end{array}$ & Разрешимая & $-1<b \leqslant 1, x_{5}$ & $y_{1}, y_{2}, y_{5}$ \\
\hline $\begin{array}{l}A_{5,71} \\
\text { Класс VI }\end{array}$ & $\begin{array}{l}{\left[e_{2}, e_{3}\right]=e_{1}} \\
{\left[e_{2}, e_{4}\right]=-e_{3}} \\
{\left[e_{3}, e_{4}\right]=e_{2}}\end{array}$ & Разрешимая & $x_{1}, 2 x_{1} x_{4}+x_{2}^{2}+x_{3}^{2}, x_{5}$ & $\begin{array}{l}y_{1}, y_{2}, \\
y_{1} y_{4}+\frac{1}{2}\left(y_{2}^{2}+\right. \\
\left.y_{3}^{2}\right), y_{5}\end{array}$ \\
\hline $\begin{array}{l}A_{5,72} \\
\text { Класс IV }\end{array}$ & $\begin{array}{l}{\left[e_{2}, e_{3}\right]=e_{1}} \\
{\left[e_{1}, e_{4}\right]=2 a e_{1}} \\
{\left[e_{2}, e_{4}\right]=a e_{2}-e_{3},} \\
{\left[e_{3}, e_{4}\right]=e_{2}+a e_{3}}\end{array}$ & Разрешимая & $a>0, x_{5}$ & $y_{1}, y_{2}, y_{5}$ \\
\hline $\begin{array}{l}A_{5,73} \\
\text { Класс II }\end{array}$ & $\begin{array}{l}{\left[e_{1}, e_{3}\right]=e_{1}} \\
{\left[e_{2}, e_{3}\right]=e_{2}} \\
{\left[e_{1}, e_{4}\right]=-e_{2}} \\
{\left[e_{2}, e_{4}\right]=e_{1}}\end{array}$ & Разрешимая & $x_{5}$ & $y_{1}, y_{2}, y_{5}$ \\
\hline
\end{tabular}

ТАБлицА 4. Все шестимерные нильпотентные вещественные алгебры Ли

\begin{tabular}{|c|c|c|c|c|c|}
\hline \multicolumn{6}{|c|}{ Неразложсимые } \\
\hline $\begin{array}{l}A_{6,1} \\
\text { Класс II }\end{array}$ & $\begin{array}{l}{\left[e_{1}, e_{2}\right]} \\
{\left[e_{1}, e_{3}\right]} \\
{\left[e_{1}, e_{5}\right]}\end{array}$ & $\begin{array}{l}=e_{3}, \\
=e_{4}, \\
=e_{6}\end{array}$ & Нильпотентная & $\begin{array}{l}x_{4}, x_{6}, x_{3} x_{6}-x_{4} x_{5} \\
2 x_{2} x_{4}-x_{3}^{2}\end{array}$ & $\begin{array}{l}y_{2}, y_{3}, y_{4}, \\
y_{5}, y_{6}\end{array}$ \\
\hline $\begin{array}{l}A_{6,2} \\
\text { Класс II }\end{array}$ & $\begin{array}{l}{\left[e_{1}, e_{2}\right]} \\
{\left[e_{1}, e_{3}\right]} \\
{\left[e_{1}, e_{4}\right]} \\
{\left[e_{1}, e_{5}\right]}\end{array}$ & $\begin{array}{l}=e_{3}, \\
=e_{4}, \\
=e_{5}, \\
=e_{6}\end{array}$ & Нильпотентная & $\begin{array}{l}x_{6}, 2 x_{4} x_{6}-x_{5}^{2}, \\
2 x_{2} x_{6}-2 x_{3} x_{5}+x_{4}^{2}, \\
3 x_{3} x_{6}^{2}-3 x_{4} x_{5} x_{6}-x_{5}^{3}\end{array}$ & $\begin{array}{l}y_{2}, y_{3}, y_{4} \\
y_{5}, y_{6}\end{array}$ \\
\hline
\end{tabular}


Таблица 4 (продолжение)

\begin{tabular}{|c|c|c|c|c|c|}
\hline $\begin{array}{l}A_{6,3} \\
\text { Класс III }\end{array}$ & $\begin{array}{l}{\left[e_{1}, e_{2}\right]} \\
{\left[e_{1}, e_{3}\right]} \\
{\left[e_{2}, e_{3}\right]}\end{array}$ & $\begin{array}{l}=e_{6} \\
=e_{4} \\
=e_{5}\end{array}$ & Нильпотентная & $\begin{array}{l}x_{4}, x_{5}, x_{6} \\
x_{1} x_{5}+x_{3} x_{6}-x_{2} x_{4}\end{array}$ & $\begin{array}{l}y_{3}, y_{4}, y_{5}, \\
y_{6}, \quad y_{2} y_{4}- \\
y_{1} y_{5}\end{array}$ \\
\hline $\begin{array}{l}A_{6,4} \\
\text { Класс II }\end{array}$ & $\begin{array}{l}{\left[e_{1}, e_{2}\right]} \\
{\left[e_{1}, e_{3}\right]} \\
{\left[e_{2}, e_{4}\right]}\end{array}$ & $\begin{array}{l}=e_{5} \\
=e_{6}, \\
=e_{6}\end{array}$ & Нильпотентная & $x_{5}, x_{6}$ & $y_{3}, y_{4}, y_{5}, y_{6}$ \\
\hline $\begin{array}{l}A_{6,5} \\
\text { Класс II }\end{array}$ & $\begin{array}{l}{\left[e_{1}, e_{3}\right]} \\
{\left[e_{1}, e_{4}\right]} \\
{\left[e_{2}, e_{3}\right]} \\
{\left[e_{2}, e_{4}\right]}\end{array}$ & $\begin{array}{l}=e_{5}, \\
=e_{6}, \\
=a e_{6}, \\
=e_{5}\end{array}$ & Нильпотентная & $a= \pm 1, x_{5}, x_{6}$ & $y_{3}, y_{4}, y_{5}, y_{6}$ \\
\hline $\begin{array}{l}A_{6,6} \\
\text { Класс II }\end{array}$ & $\begin{array}{l}{\left[e_{1}, e_{2}\right]} \\
{\left[e_{1}, e_{3}\right]} \\
{\left[e_{1}, e_{4}\right]} \\
{\left[e_{2}, e_{3}\right]}\end{array}$ & $\begin{array}{l}=e_{6}, \\
=e_{4}, \\
=e_{5}, \\
=e_{5}\end{array}$ & Нильпотентная & $x_{5}, x_{6}$ & $y_{3}, y_{4}, y_{5}, y_{6}$ \\
\hline $\begin{array}{l}A_{6,7} \\
\text { Класс II }\end{array}$ & $\begin{array}{l}{\left[e_{1}, e_{3}\right]} \\
{\left[e_{1}, e_{4}\right]} \\
{\left[e_{2}, e_{3}\right]}\end{array}$ & $\begin{array}{l}=e_{4} \\
=e_{5} \\
=e_{6}\end{array}$ & Нильпотентная & $x_{5}, x_{6}$ & $y_{3}, y_{4}, y_{5}, y_{6}$ \\
\hline $\begin{array}{l}A_{6,8} \\
\text { Класс II }\end{array}$ & $\begin{array}{l}{\left[e_{1}, e_{2}\right]} \\
{\left[e_{1}, e_{3}\right]} \\
{\left[e_{2}, e_{5}\right]}\end{array}$ & $\begin{array}{l}=e_{3}+e_{5} \\
=e_{4} \\
=e_{6}\end{array}$ & Нильпотентная & $x_{4}, x_{6}$ & $y_{3}, y_{4}, y_{5}, y_{6}$ \\
\hline $\begin{array}{l}A_{6,9} \\
\text { Класс II }\end{array}$ & $\begin{array}{l}{\left[e_{1}, e_{2}\right]} \\
{\left[e_{1}, e_{3}\right]} \\
{\left[e_{1}, e_{5}\right]} \\
{\left[e_{2}, e_{3}\right]}\end{array}$ & $\begin{array}{l}=e_{3}, \\
=e_{4} \\
=e_{6} \\
=e_{6}\end{array}$ & Нильпотентная & $x_{4}, x_{6}$ & $y_{3}, y_{4}, y_{5}, y_{6}$ \\
\hline $\begin{array}{l}A_{6,10} \\
\text { Класс II }\end{array}$ & $\begin{array}{l}{\left[e_{1}, e_{2}\right]} \\
{\left[e_{1}, e_{3}\right]} \\
{\left[e_{1}, e_{4}\right]} \\
{\left[e_{2}, e_{3}\right]} \\
{\left[e_{2}, e_{4}\right]}\end{array}$ & $\begin{array}{l}=e_{3}, \\
=e_{5}, \\
=e_{6}, \\
=a e_{6}, \\
=e_{5}\end{array}$ & Нильпотентная & $a= \pm 1, x_{5}, x_{6}$ & $y_{3}, y_{4}, y_{5}, y_{6}$ \\
\hline $\begin{array}{l}A_{6,11} \\
\text { Класс II }\end{array}$ & $\begin{array}{l}{\left[e_{1}, e_{2}\right]} \\
{\left[e_{1}, e_{3}\right]} \\
{\left[e_{1}, e_{4}\right]} \\
{\left[e_{2}, e_{3}\right]}\end{array}$ & $\begin{array}{l}=e_{3}, \\
=e_{4}, \\
=e_{5} \\
=e_{6}\end{array}$ & Нильпотентная & $x_{5}, x_{6}$ & $y_{3}, y_{4}, y_{5}, y_{6}$ \\
\hline $\begin{array}{l}A_{6,12} \\
\text { Класс II }\end{array}$ & $\begin{array}{l}{\left[e_{1}, e_{3}\right]} \\
{\left[e_{1}, e_{4}\right]} \\
{\left[e_{2}, e_{5}\right]}\end{array}$ & $\begin{array}{l}=e_{4} \\
=e_{6} \\
=e_{6}\end{array}$ & Нильпотентная & $x_{6}, 2 x_{3} x_{6}-x_{4}^{2}$ & $y_{3}, y_{4}, y_{5}, y_{6}$ \\
\hline $\begin{array}{l}A_{6,13} \\
\text { Класс II }\end{array}$ & $\begin{array}{l}{\left[e_{1}, e_{2}\right]} \\
{\left[e_{1}, e_{3}\right]} \\
{\left[e_{1}, e_{4}\right]} \\
{\left[e_{2}, e_{5}\right]}\end{array}$ & $\begin{array}{l}=e_{5}, \\
=e_{4}, \\
=e_{6}, \\
=e_{6}\end{array}$ & Нильпотентная & $x_{6}, 2 x_{3} x_{6}-x_{4}^{2}$ & $y_{3}, y_{4}, y_{5}, y_{6}$ \\
\hline $\begin{array}{l}A_{6,14} \\
\text { Класс II }\end{array}$ & $\begin{array}{l}{\left[e_{1}, e_{3}\right]} \\
{\left[e_{1}, e_{4}\right]} \\
{\left[e_{2}, e_{3}\right]} \\
{\left[e_{2}, e_{5}\right]}\end{array}$ & $\begin{array}{l}=e_{4} \\
=e_{6} \\
=e_{5} \\
=a e_{6}\end{array}$ & Нильпотентная & $a \neq 0, x_{6}, x_{5}^{2}+a x_{4}^{2}-2 a x_{3} x_{6}$ & $y_{3}, y_{4}, y_{5}, y_{6}$ \\
\hline $\begin{array}{l}A_{6,15} \\
\text { Класс II }\end{array}$ & $\begin{array}{l}{\left[e_{1}, e_{2}\right]} \\
{\left[e_{1}, e_{3}\right]} \\
{\left[e_{1}, e_{4}\right]} \\
{\left[e_{2}, e_{5}\right]}\end{array}$ & $\begin{array}{l}=e_{3}+e_{5} \\
=e_{4} \\
=e_{6} \\
=e_{6}\end{array}$ & Нильпотентная & $x_{6}, x_{4}^{2}-2 x_{3} x_{6}$ & $y_{3}, y_{4}, y_{5}, y_{6}$ \\
\hline $\begin{array}{l}A_{6,16} \\
\text { Класс II }\end{array}$ & $\begin{array}{l}{\left[e_{1}, e_{3}\right]} \\
{\left[e_{1}, e_{4}\right]} \\
{\left[e_{1}, e_{5}\right]} \\
{\left[e_{2}, e_{3}\right]} \\
{\left[e_{2}, e_{4}\right]}\end{array}$ & $\begin{array}{l}=e_{4} \\
=e_{5} \\
=e_{6} \\
=e_{5} \\
=e_{6}\end{array}$ & Нильпотентная & $x_{6}, 3 x_{3} x_{6}^{2}+x_{5}^{3}-3 x_{4} x_{5} x_{6}$ & $y_{3}, y_{4}, y_{5}, y_{6}$ \\
\hline
\end{tabular}


Таблица 4 (продолжение)

\begin{tabular}{|c|c|c|c|c|c|}
\hline $\begin{array}{l}A_{6,17} \\
\text { Класс II }\end{array}$ & $\begin{array}{l}{\left[e_{1}, e_{2}\right]} \\
{\left[e_{1}, e_{3}\right]} \\
{\left[e_{1}, e_{4}\right]} \\
{\left[e_{2}, e_{5}\right]}\end{array}$ & $\begin{array}{l}=e_{3} \\
=e_{4} \\
=e_{6} \\
=e_{6}\end{array}$ & Нильпотентная & $x_{6}, x_{4}^{2}-2 x_{3} x_{6}$ & $y_{3}, y_{4}, y_{5}, y_{6}$ \\
\hline $\begin{array}{l}A_{6,18} \\
\text { Класс II }\end{array}$ & $\begin{array}{l}{\left[e_{1}, e_{2}\right]} \\
{\left[e_{1}, e_{3}\right]} \\
{\left[e_{1}, e_{4}\right]} \\
{\left[e_{2}, e_{3}\right]} \\
{\left[e_{2}, e_{5}\right]}\end{array}$ & $\begin{array}{l}=e_{3}, \\
=e_{4}, \\
=e_{6}, \\
=e_{5}, \\
=a e_{6}\end{array}$ & Нильпотентная & $a \neq 0, x_{6}, x_{5}^{2}+a x_{4}^{2}-2 a x_{3} x_{6}$ & $y_{3}, y_{4}, y_{5}, y_{6}$ \\
\hline $\begin{array}{l}A_{6,19} \\
\text { Класс II }\end{array}$ & $\begin{array}{l}{\left[e_{1}, e_{2}\right]} \\
{\left[e_{1}, e_{3}\right]} \\
{\left[e_{1}, e_{4}\right]} \\
{\left[e_{1}, e_{5}\right]} \\
{\left[e_{2}, e_{3}\right]}\end{array}$ & $\begin{array}{l}=e_{3}, \\
=e_{4}, \\
=e_{5}, \\
=e_{6}, \\
=e_{6}\end{array}$ & Нильпотентная & $x_{6}, x_{5}^{2}-2 x_{4} x_{6}$ & $y_{3}, y_{4}, y_{5}, y_{6}$ \\
\hline $\begin{array}{l}A_{6,20} \\
\text { Класс II }\end{array}$ & $\begin{array}{l}{\left[e_{1}, e_{2}\right]} \\
{\left[e_{1}, e_{3}\right]} \\
{\left[e_{1}, e_{4}\right]} \\
{\left[e_{1}, e_{5}\right]} \\
{\left[e_{2}, e_{3}\right]} \\
{\left[e_{2}, e_{4}\right]}\end{array}$ & $\begin{array}{l}=e_{3}, \\
=e_{4}, \\
=e_{5}, \\
=e_{6}, \\
=e_{5}, \\
=e_{6}\end{array}$ & Нильпотентная & $x_{6}, x_{5}^{3}+3 x_{3} x_{6}^{2}-3 x_{4} x_{5} x_{6}$ & $y_{3}, y_{4}, y_{5}, y_{6}$ \\
\hline $\begin{array}{l}A_{6,21} \\
\text { Класс III }\end{array}$ & $\begin{array}{l}{\left[e_{1}, e_{2}\right]} \\
{\left[e_{1}, e_{5}\right]} \\
{\left[e_{2}, e_{3}\right]} \\
{\left[e_{2}, e_{4}\right]} \\
{\left[e_{3}, e_{4}\right]}\end{array}$ & $\begin{array}{l}=e_{3}, \\
=e_{6}, \\
=e_{4}, \\
=e_{5}, \\
=e_{6}\end{array}$ & Нильпотентная & $x_{6}, x_{4}^{2}+2 x_{2} x_{6}-2 x_{3} x_{5}$ & $\begin{array}{l}y_{4}, y_{5}, y_{6} \\
y_{2} y_{6}-y_{3} y_{5}\end{array}$ \\
\hline $\begin{array}{l}A_{6,22} \\
\text { Класс III }\end{array}$ & $\begin{array}{l}{\left[e_{1}, e_{2}\right]} \\
{\left[e_{1}, e_{3}\right]} \\
{\left[e_{1}, e_{5}\right]} \\
{\left[e_{2}, e_{3}\right]} \\
{\left[e_{2}, e_{4}\right]} \\
{\left[e_{3}, e_{4}\right]}\end{array}$ & $\begin{array}{l}=e_{3}, \\
=e_{5}, \\
=e_{6}, \\
=e_{4}, \\
=e_{5}, \\
=e_{6}\end{array}$ & Нильпотентная & $\begin{array}{l}x_{6}, 2 x_{5}^{3}+3 x_{4}^{2} x_{6}-6 x_{2} x_{6}^{2}- \\
6 x_{3} x_{5} x_{6}\end{array}$ & $\begin{array}{l}y_{4}, y_{5}, y_{6} \\
y_{2} y_{6}-y_{3} y_{5}\end{array}$ \\
\hline \multicolumn{6}{|c|}{ Разложсимые } \\
\hline $\begin{array}{l}A_{6,23} \\
\text { Класс II }\end{array}$ & - & & Абелева & $x_{1}, x_{2}, x_{3}, x_{4}, x_{5}, x_{6}$ & $\begin{array}{l}y_{1}, y_{2}, y_{3} \\
y_{4}, y_{5}, y_{6}\end{array}$ \\
\hline $\begin{array}{l}A_{6,24} \\
\text { Класс II }\end{array}$ & {$\left[e_{1}, e_{2}\right]$} & $=e_{3}$ & Нильпотентная & $x_{3}, x_{4}, x_{5}, x_{6}$ & $\begin{array}{l}y_{2}, y_{3}, y_{4} \\
y_{5}, y_{6}\end{array}$ \\
\hline $\begin{array}{l}A_{6,25} \\
\text { Класс II }\end{array}$ & $\begin{array}{l}{\left[e_{1}, e_{2}\right]} \\
{\left[e_{4}, e_{5}\right]}\end{array}$ & $\begin{array}{l}=e_{3} \\
=e_{6}\end{array}$ & Нильпотентная & $x_{3}, x_{6}$ & $y_{2}, y_{3}, y_{5}, y_{6}$ \\
\hline $\begin{array}{l}A_{6,26} \\
\text { Класс II }\end{array}$ & $\begin{array}{l}{\left[e_{1}, e_{2}\right]} \\
{\left[e_{1}, e_{3}\right]}\end{array}$ & $\begin{array}{l}=e_{3} \\
=e_{4}\end{array}$ & Нильпотентная & $x_{3}^{2}-2 x_{2} x_{4}, x_{4}, x_{5}, x_{6}$ & $\begin{array}{l}y_{2}, y_{3}, y_{4} \\
y_{5}, y_{6}\end{array}$ \\
\hline $\begin{array}{l}A_{6,27} \\
\text { Класс II }\end{array}$ & $\begin{array}{l}{\left[e_{3}, e_{5}\right]} \\
{\left[e_{4}, e_{5}\right]}\end{array}$ & $\begin{array}{l}=e_{1} \\
=e_{2}\end{array}$ & Нильпотентная & $x_{1}, x_{2}, x_{2} x_{3}-x_{1} x_{4}, x_{6}$ & $\begin{array}{l}y_{1}, y_{2}, y_{3} \\
y_{4}, y_{6}\end{array}$ \\
\hline $\begin{array}{l}A_{6,28} \\
\text { Класс II }\end{array}$ & $\begin{array}{l}{\left[e_{2}, e_{5}\right]} \\
{\left[e_{3}, e_{5}\right]} \\
{\left[e_{4}, e_{5}\right]}\end{array}$ & $\begin{array}{l}=e_{1} \\
=e_{2} \\
=e_{3}\end{array}$ & Нильпотентная & $\begin{array}{l}x_{1}, x_{2}^{2}-2 x_{1} x_{3}, \\
x_{2}^{3}+3 x_{1}^{2} x_{4}-3 x_{1} x_{2} x_{3}, x_{6}\end{array}$ & $\begin{array}{l}y_{1}, y_{2}, y_{3} \\
y_{4}, y_{6}\end{array}$ \\
\hline $\begin{array}{l}A_{6,29} \\
\text { Класс III }\end{array}$ & $\begin{array}{l}{\left[e_{3}, e_{4}\right]} \\
{\left[e_{3}, e_{5}\right]} \\
{\left[e_{4}, e_{5}\right]}\end{array}$ & $\begin{array}{l}=e_{2} \\
=e_{1} \\
=e_{3}\end{array}$ & Нильпотентная & $\begin{array}{l}x_{1}, x_{2}, \\
x_{3}^{2}+2 x_{2} x_{5}-2 x_{1} x_{4}, x_{6}\end{array}$ & $\begin{array}{l}y_{1}, y_{2}, y_{3}, \\
y_{1} y_{4}-y_{2} y_{5} \\
y_{6}\end{array}$ \\
\hline $\begin{array}{l}A_{6,30} \\
\text { Класс II }\end{array}$ & $\begin{array}{l}{\left[e_{2}, e_{4}\right]} \\
{\left[e_{3}, e_{5}\right]}\end{array}$ & $\begin{array}{l}=e_{1} \\
=e_{1}\end{array}$ & Нильпотентная & $x_{1}, x_{6}$ & $y_{1}, y_{2}, y_{3}, y_{6}$ \\
\hline
\end{tabular}




\section{Таблица 4 (продолжение)}

\begin{tabular}{|l|l|l|l|l|}
\hline $\begin{array}{l}A_{6,31} \\
\text { Класс II }\end{array}$ & $\begin{array}{l}{\left[e_{3}, e_{4}\right]=e_{1},} \\
{\left[e_{2}, e_{5}\right]=e_{1},} \\
{\left[e_{3}, e_{5}\right]=e_{2}}\end{array}$ & Нильпотентная & $x_{1}, x_{6}$ & $y_{1}, y_{2}, y_{3}, y_{6}$ \\
\hline \multirow{3}{*}{$A_{6,32}$} & $\begin{array}{l}{\left[e_{3}, e_{4}\right]=e_{1},} \\
\text { Kласс II }\end{array}$ & & & \\
& {$\left[e_{2}, e_{5}\right]=e_{1}$,} \\
{$\left[e_{3}, e_{5}\right]=e_{2}$,} & Нильпотентная & $x_{1}, x_{6}$ & $y_{1}, y_{2}, y_{3}, y_{6}$ \\
\hline$\left.e_{4}, e_{5}\right]=e_{3}$ & & & \\
\hline
\end{tabular}

\section{Список литературы}

[1] В. В. Трофимов, А. Т. Фоменко, Алгебра и геометрия интегрируемых гамильтоновых дифференииальных уравнений, Факториал, М.; Просперус, Ижевск, 1995.

[2] А. С. Мищенко, А. Т. Фоменко, "Уравнения Эйлера на конечномерных группах Ли", Изв. АН СССР. Сер. матем., 42:2 (1978), 396-415; англ. пер.: А. S. Miščenko, A. T. Fomenko, "Euler equations on finite-dimensional Lie groups", Math. USSR-Izv., 12:2 (1978), 371-389.

[3] А. С. Мищенко, А. Т. Фоменко, “Обобщенный метод Лиувилля интегрирования гамильтоновых систем", Функи. анализ и его прил., 12:2 (1978), 46-56; англ. пер.: A. S. Mishchenko, A. T. Fomenko, "Generalized Liouville method of integration of Hamiltonian systems", Funct. Anal. Appl., 12:2 (1978), 113-121.

[4] А. С. Мищенко, А. Т. Фоменко, "Некоммутативное интегрирование гамильтоновых систем и его приложения", Механика твердого тела, 1978, № 4, 187-188.

[5] А. С. Мищенко, А.Т. Фоменко, "Интегрирование уравнений Эйлера на полупростых алгебрах Ли", Докл. АН СССР, 231:3 (1976), 536-538; англ. пер.: A.S. Mishchenko, A.T. Fomenko, "On the integration of the Euler equations on semisimple Lie algebras", Soviet Math. Dokl., 17:6 (1976), 1591-1594.

[6] А. С. Мищенко, А. Т. Фоменко, "Интегрируемость уравнений Эйлера на полупростых алгебрах Ли", Тр. сем. по вект. и тенз. анализу, 19, Изд-во Моск. ун-та, М., 1979, 3-94; англ. пер.: А. S. Mishchenko, А. T. Fomenko, "Integrability of Euler equations on semisimple Lie algebras", Selecta Math. Soviet., 2:3 (1982), 207-291.

[7] В. В. Трофимов, "Уравнения Эйлера на борелевских подалгебрах полупростых алгебр Ли", Изв. АН СССР. Сер. матем., 43:3 (1979), 714-732; англ. пер.: V.V. Trofimov, "Euler equations on Borel subalgebras of semisimple Lie algebras", Math. USSR-Izv., 14:3 (1980), 653-670.

[8] В.В. Трофимов, А.Т. Фоменко, “Динамические системы на орбитах линейных представлений групп Ли и полная интегрируемость некоторых гидродинамических систем", Функи. анализ и его прил., 17:1 (1983), 31-39; англ. пер.: V.V. Trofimov, A.T. Fomenko, "Dynamical systems on the orbits of linear representations of Lie groups and the complete integrability of certain hydrodynamical systems", Funct. Anal. Appl., 17:1 (1983), 23-29.

[9] В. В. Трофимов, А. Т. Фоменко, "Интегрируемость по Лиувиллю гамильтоновых систем на алгебрах Ли", УМH, 39:2 (1984), 3-56; англ. пер.: V. V. Trofimov, A. T. Fomenko, "Liouville integrability of Hamiltonian systems on Lie algebras", Russian Math. Surveys, 39:2 (1984), 1-67.

[10] А. В. Браилов, А. Т. Фоменко, "Топология интегральных подмногообразий вполне интегрируемых гамильтоновых систем", Матем. сб., 134(176):3(11) (1987), 375-385; англ. пер.: V. V. Trofimov, A. T. Fomenko, "The topology of integral submanifolds of completely integrable Hamiltonian systems", Math. USSR-Sb., 62:2 (1989), 373-383. 
[11] С. Т. Садэтов, "Доказательство гипотезы Мищенко-Фоменко", Докл. РАН, 397:6 (2004), 751-754; англ. пер.: S. T. Sadetov, "A proof of the Mishchenko-Fomenko conjecture", Dokl. Math., 70:1 (2004), 635-638.

[12] А. В. Болсинов, "Полные инволютивные наборы полиномов в пуассоновых алгебрах: доказательство гипотезы Мищенко-Фоменко", Тр. сем. по вект. и тенз. анализу, 26, Изд-во Моск. ун-та, М., 2005, 87-109.

[13] J. Patera, R. T. Sharp, P. Winternitz, H. Zassenhaus, "Invariants of real low dimension Lie algebras", J. Mathematical Phys., 17:6 (1976), 986-994.

[14] В.В. Морозов, "Классификация нильпотентных алгебр Ли шестого порядка", Изв. вузов. Матем., 1958, № 4, 161-171.

[15] Г. М. Мубаракзянов, "Классификация вещественных структур алгебр Ли пятого порядка", Изв. вузов. Матем., 1963, № 3, 99-106.

[16] Ю.С. Балина, Коприсоединенное представление и инварианты групп Ли мальх размерностей, Дипломная работа, МГУ им. М. В. Ломоносова, мех.-мат. ф-т, каф. Дифф. геометрии и приложений, М., 2002.

[17] К. М. Зуев, Формальный метод сдвига аргумента и геометрия интегрируемых геодезических потоков, Дис. ... канд. физ.-матем. наук, МГУ им. М. В. Ломоносова, М., 2008.

\author{
А. А. Короткевич (А. А. Korotkevich) \\ Механико-математический факультет \\ Московского государственного университета \\ им. М. В. Ломоносова \\ E-mail: korotkevich_aa@mail.ru
}

Поступила в редакцию

13.03.2009 